\title{
Gauge fixing, BRS invariance and Ward identities for randomly stirred flows
}

\author{
Arjun Berera ${ }^{1, *}$ and David Hochberg ${ }^{2,+}$ \\ ${ }^{1}$ School of Physics and Astronomy, \\ University of Edinburgh, Edinburgh, EH9 3JZ, U.K \\ ${ }^{2}$ Centro de Astrobiología (CSIC-INTA), Ctra. Ajalvir Km. 4, \\ 28850 Torrejón de Ardoz, Madrid, Spain
}

(Dated: November 3, 2018)

\begin{abstract}
The Galilean invariance of the Navier-Stokes equation is shown to be akin to a global gauge symmetry familiar from quantum field theory. This symmetry leads to a multiple counting of infinitely many inertial reference frames in the path integral approach to randomly stirred fluids. This problem is solved by fixing the gauge, i.e., singling out one reference frame. The gauge fixed theory has an underlying Becchi-Rouet-Stora (BRS) symmetry which leads to the Ward identity relating the exact inverse response and vertex functions. This identification of Galilean invariance as a gauge symmetry is explored in detail, for different gauge choices and by performing a rigorous examination of a discretized version of the theory. The Navier-Stokes equation is also invariant under arbitrary rectilinear frame accelerations, known as extended Galilean invariance (EGI). We gauge fix this extended symmetry and derive the generalized Ward identity that follows from the BRS invariance of the gauge-fixed theory. This new Ward identity reduces to the standard one in the limit of zero acceleration. This gauge-fixing approach unambiguously shows that Galilean invariance and EGI constrain only the zero mode of the vertex but none of the higher wavenumber modes.
\end{abstract}

PACS numbers: 47.27.ef, 11.10.-z, 03.50.-z

*Electronic address: ab@ph.ed.ac.uk

$\dagger$ Electronic address: hochbergd@inta.es 


\section{INTRODUCTION}

The formulation of the Navier Stokes equation with random forcing as a classical stochastic field theory [1, 2, 3, 4, 5, 6, 7] opens up the way to apply the methods originally developed for quantum fields [8]. This fact has been used in the majority of cases for applying renormalization group (RG) methods to models of fully developed turbulence [9]. This field theory approach has also been used on many occasions to derive exact relations between different correlation functions implied by Galilean invariance. These are akin to the Ward-Takahashi identities (WTI) of quantum field theory [10]. The most well known of these exact identities relates the vertex and response functions [2]. Based on this relation, claims have been made concerning the non-renormalization, under the renormalization group, of the advective or inertial term in the Navier-Stokes equation [2, 3, 4, ㄴ, 7, 11, 12, 13]. Despite the long history of applying these field theoretic methods, two major problems connected with the Galilean invariance of the path integral framework of randomly stirred fluids have been brought to light recently. The first problem has to do with the functional itself. The standard dynamic functional for the randomly forced Navier-Stokes equation leads to spurious relations for the correlation functions involving the zero-mode of the fluid velocity. This dynamic functional is therefore ill-defined. As shown in [13] the dynamic functional for the randomly stirred Navier Stokes equation can be regarded as a gauge theory, and then the problem of the spurious relations is solved by fixing the gauge, which thus breaks the Galilean invariance. This is a new and important observation and leads to a well-defined functional expression, which has been missing for the past three decades. However, once the gauge is fixed, it is crucial to verify whether Galilean invariance (GI) can be restored, and this is achieved by recognizing that the gauge-fixed theory is BRS invariant. This brings us to the second problem which has to do with the past mis-interpretations of the Ward identity that follows from the functional. This BRS invariance leads to a Ward identity whose physical consequence is that only the zero mode part of the full vertex, but none of the higher spatial modes, is constrained. In other words, contributions from the non-zero modes to the vertex can receive corrections under renormalization. This conclusion therefore provides greater clarity to, and in some cases goes against, previous interpretations regarding the physical consequences of GI for the vertex in the Navier-Stokes equation [2, 3, 4, 6, 7, 11, 12, 13].

The present work supports the conclusions drawn in [7] in [12] and in [13], and develops 
in full detail the results presented in [13], while also generalizing that work. For this, the gauge fixing concept for the NSE is explored in detail including the treatment of distinct gauge choices and the associated implications for the BRS symmetry left in the theory. Also, the analogy between the Ward identities found here for the NSE and those in QED is carefully examined. Finally, the considerations developed so far for Galilean invariance are thoroughly worked out here for extended Galilean invariance, which is a more general global symmetry of the NSE. We derive the consequences of this higher symmetry which leads to a new Ward identity for the forced NSE. The Ward identity for EGI has not to our knowledge been derived nor applied to the Navier-Stokes equation before. We then use it to see how the convective term in the Navier Stokes equation can renormalize. This has direct physical consequences. For this case, the time dependent but spatial zero mode part of the vertex is related to viscosity and mass renormalizations, while none of the higher spatial modes are constrained by EGI. These facts constitute the physical results of this paper.

In order to consider the consequences of breaking Galilean invariance via gauge fixing, we start with the ensemble of histories of Navier-Stokes dynamics, and make direct use of the standard functional integral methods. The dynamic generating functional for a randomly stirred incompressible fluid has been extensively studied for many years [1, 2, 3, 4, 5, 6, 17]. It is based on the path integral approach [1, 15, 16, 17] to classical statistical dynamics [18] and is given by

$$
Z=\int[D \mathbf{V}][D \boldsymbol{\sigma}] \exp \{-S[\mathbf{V}, \boldsymbol{\sigma}]\}
$$

where the action is ${ }^{1}$

$$
\begin{aligned}
S[\mathbf{V}, \boldsymbol{\sigma}]= & \frac{1}{2} \int d \mathbf{x} d t \int d \mathbf{y} \sigma_{i}(\mathbf{x}, t) D_{i j}(\mathbf{x}-\mathbf{y}) \sigma_{j}(\mathbf{y}, t) \\
& -i \int d \mathbf{x} d t \sigma_{k}\left(\frac{\partial V_{k}}{\partial t}+P_{k j}(\nabla) \frac{\partial\left(V_{l} V_{j}\right)}{\partial x_{l}}-\nu_{0} \nabla^{2} V_{k}\right) .
\end{aligned}
$$

The instantaneous fluid velocity is $\mathbf{V}$, the conjugate field is $\boldsymbol{\sigma} ; D_{i j}(\mathbf{x})$ is the only nonvanishing cumulant of the stationary stirring force and $\mathbf{x}$ is a coordinate vector in $d$ dimensions. The projection operator is $P_{i j}(\nabla)=\left(\delta_{i j}-\nabla_{i} \frac{1}{\nabla^{2}} \nabla_{j}\right)$ and $\nu_{0}$ denotes the bare

\footnotetext{
${ }^{1}$ A Martin-Siggia-Rose type action can be derived for general Langevin equations of motion [19], and this includes the Navier-Stokes equation (NSE) with random forcing. The Jacobian that arises in passing from the general stochastic equation to the path integral can, in effect, always be set to unity. This is treated in detail in Ref. [19]. See also Ref. [17] and Refs. [4, 5] which prove that this Jacobian is unity for the particular case of the forced NSE.
} 
(unrenormalized) fluid viscosity. Now consider a second primed frame moving with a constant velocity $\mathbf{c}$ with respect to the unprimed frame. Then the relations between the instantaneous fluid velocity, conjugate field, time and coordinates of events in both frames are given by:

$$
\begin{aligned}
\mathbf{V}^{\mathbf{c}}(\mathbf{x}, t) & =\mathbf{V}^{\prime}\left(\mathbf{x}^{\prime}, t^{\prime}\right)+\mathbf{c} \\
\boldsymbol{\sigma}^{c}(\mathbf{x}, t) & =\boldsymbol{\sigma}^{\prime}\left(\mathbf{x}^{\prime}, t^{\prime}\right) \\
t & =t^{\prime} \\
\mathbf{x} & =\mathbf{x}^{\prime}+\mathbf{c} t
\end{aligned}
$$

$\mathbf{V}^{\mathbf{c}}$ denotes the result of the Galilean transformation applied to the velocity field $\mathbf{V}$, and similarly for $\boldsymbol{\sigma}^{\boldsymbol{c}}$. Due to the Galilean invariance of the measure $\left[D \mathbf{V}^{\mathbf{c}}\right]\left[D \boldsymbol{\sigma}^{c}\right]=\left[D \mathbf{V}^{\prime}\right]\left[D \boldsymbol{\sigma}^{\prime}\right]^{2}$ and the action $S$ under the transformation in Eqs.(3) 6 ), it is clear that the generating functional Eq.(1) sums over all fluid velocity configurations. This results in multiple counting of physically equivalent configurations: namely, those that are equivalent up to a Galilean transformation (GT). In other words, this integral includes the sum over all inertial reference frames. This feature leads to spurious relations among velocity correlation functions which must be removed in order to render a well defined generating functional for the theory [13]. The solution to this problem was demonstrated to be provided by gauge fixing [13]. Moreover, if one wants to use the path integral for the Navier-Stokes equation for computing non-Galilean invariant quantities, like $n$-point velocity correlation functions, it is essential to carry out a gauge fixing along the lines we present here, and this is especially true for numerical implementations [14]. Gauge fixing for $Z$ can be viewed operationally in exactly the same way as in quantum field theory. In the present context, we must divide velocity configuration space into equivalence classes called the "orbits" of the GT. An orbit of the GT includes all velocity field configurations which result when all possible GT's are applied to a given initial velocity field configuration. Thus, $Z$ is proportional to the infinite volume of these orbits, and this volume factor is extracted out before defining this functional. However, once the Galilean invariance is explicitly broken, it is crucially important to verify

\footnotetext{
2 The change of variables Eq.(3) in the functional measure generates a unit jacobian: $\mathcal{J}=\operatorname{det} \frac{\delta V_{i}(\mathbf{x}, t)}{\delta V_{j}^{\prime}\left(\mathbf{y}^{\prime}, \tau^{\prime}\right)}=$ $\operatorname{det} \frac{\delta\left(V_{i}^{\prime}\left(\mathbf{x}^{\prime}, t^{\prime}\right)+c_{i}\right)}{\delta V_{j}^{\prime}\left(\mathbf{y}^{\prime}, \tau^{\prime}\right)}=\operatorname{det}\left(\delta_{i j} \delta\left(\mathbf{x}^{\prime}-\mathbf{y}^{\prime}\right) \delta\left(\tau^{\prime}-t^{\prime}\right)\right)$. Similarly for the transformation Eq. (4) of the conjugate field. The Jacobian is also unity for the extended Galilean transformation in Eqs.(44.47) and Eq.(49).
} 
whether the exact identities relating inverse response and vertex functions continue to hold. We demonstrate that this is the case, because the resultant gauge fixed functional has an underlying Becchi-Rouet-Stora (BRS) symmetry. This symmetry leads to exact identities structurally similar to the Slavnov-Taylor (ST) identities of quantum field theory [20]. Here, the Slavnov-Taylor identity is significant because the ghost degrees of freedom make absolutely explicit that the vertex nonrenormalization only affects the zero mode. Contrary to previous assertions and claims, it is not even in the $k \rightarrow 0$ limit that the claimed vertex nonrenormalization holds, but rather only at $k=0$, the zero-mode. This is a subtle but very important point that must be born in mind.

The outline of this paper is as follows. In Sec. II A we pick a gauge which singles out a unique inertial reference frame, and carry out the gauge fixing procedure for the dynamic functional in Eq. (1). This leads to a gauge fixed action. In Sec. IIB we write down the Becchi-Rouet-Stora (BRS) transformation that leaves this gauge fixed action invariant. This BRS invariance leads to the Slavnov-Taylor identities for the dynamic functional. These in turn imply the crucial Ward identity for the effective action that has been derived previously on numerous occasions, but always derived for the non-gauge fixed functional [2, 3, 4, 6, 7, 11]. In Sec IC we prove that this crucial identity remains valid, even after explicitly breaking the Galilean invariance, and selecting a fixed reference frame. Other gauge choices are possible, as illustrated in Sec IID. The stochastic field theory for the NSE can be regularized in a space-time box and a corresponding Ward identity is derived in Sec IIE. This is used to demonstrate that the spurious relations first found in [13] for the continuum field theory arise in the regularized theory as well, and are therefore not an artifact of the continuum limit. The Navier-Stokes equation is also invariant under arbitrary rectilinear frame accelerations, a symmetry known as extended Galilean invariance (EGI) [21]. This is reviewed briefly in Sec ПIIA. Gauge fixing is carried out in Sec IIIB by choosing a single accelerating frame and the BRS transformation that leaves the corresponding gauge fixed action invariant is written down in Sec IIIC. To complete the analysis, we derive the Slavnov-Taylor identities for EGI in Sec IIID and show that these lead to a new identity for the inverse response and vertex functions. This identity reduces to the standard one in the limit of vanishing reference frame accelerations. The Ward identities that follow from the EGI and GI in stochastic flows bear a striking resemblance to the well known QED Ward identities that follow from the local U(1) gauge invariance. The similarities 
as well as the differences are spelled out in Sec IV. We discuss the implications of both Galilean and extended Galilean invariance for vertex renormalization in Sec $\mathrm{V}$ and present our conclusions in Sec VI. The main steps for gauge fixing the Galilean invariant dynamic functional are collected in an Appendix which closely parallel the standard technique as employed in quantum field theory.

\section{GALILEAN INVARIANCE: GAUGE FIXING AND BECCHI-ROUET-STORA SYMMETRY}

\section{A. The $\mathbf{V}_{0}^{2}$ gauge}

For the reasons stated above, we proceed to gauge-fix the dynamic functional in Eq.(1). The basic outline for doing so in an arbitrary gauge $\mathbf{f}(\mathbf{V})$ is summarized in general terms in the Appendix A. This is intended to parallel closely the procedure as it is used in quantum gauge theories. Once we choose the specific gauge introduced below, we then only have to calculate the corresponding Fadeev-Popov determinant Eq.(A5), and then select the arbitrary function $\mathbf{U}$ that appears in Eq.(A6 to complete the procedure. Here and below, we consider spatially bounded fluid systems.

Recall that the purpose of gauge fixing here is to single out one inertial reference frame. This can be accomplished by constraining the zero mode part of the full instantaneous velocity field, because this mode corresponds to the constant velocity of the system as a whole, that is, its bulk velocity. We thus make the gauge choice specified by

$$
\mathbf{f}(\mathbf{V})=\frac{1}{v o l} \int d \mathbf{x} d t \mathbf{V}(\mathbf{x}, t)
$$

where $v o l=L^{3} T$ is the (finite) volume of the space-time box bounding the fluid. This projects out the zero mode from the full instantaneous velocity field $\mathbf{V}$, as required.

Next, from Eqs.(3,5,6), we have

$$
\begin{aligned}
V_{j}^{\mathbf{c}}(\mathbf{x}, t) & =V_{j}^{\prime}(\mathbf{x}-\mathbf{c} t, t)+c_{j}, \\
& =V_{j}^{\prime}(\mathbf{x}, t)-c_{k} t \frac{\partial V_{j}^{\prime}(\mathbf{x}, t)}{\partial x_{k}}+c_{j}+O\left(c^{2}\right), \\
\delta V_{j}^{\mathbf{c}}(\mathbf{x}, t) & =V_{j}^{\prime}-V_{j}^{\mathbf{c}}=c_{k} t \frac{\partial V_{j}^{\prime}(\mathbf{x}, t)}{\partial x_{k}}-c_{j}, \\
\text { Thus } \quad \frac{\partial V_{j}^{\mathbf{c}}(\mathbf{x}, t)}{\partial c_{m}}\left\lfloor_{\mathbf{c}=\mathbf{0}}\right. & =t \frac{\partial V_{j}^{\prime}(\mathbf{x}, t)}{\partial x_{m}}-\delta_{j m} .
\end{aligned}
$$


Then from Eqs.(7],8) we have

$$
\left.\left(\frac{\partial f_{i}}{\partial c_{j}}\right)\right|_{\mathbf{c}=\mathbf{0}}=\frac{1}{v o l} \int d \mathbf{x} d t\left(t \frac{\partial V_{i}^{\prime}(\mathbf{x}, t)}{\partial x_{j}}-\delta_{i j}\right) .
$$

This simplifies due to boundary conditions. Imposing the physically reasonable periodic boundary conditions for a spatial box of side length $L$, we have

$$
\int_{0}^{L} d x_{j} \frac{\partial V_{i}(\mathbf{x}, t)}{\partial x_{j}}=V_{i}\left(\mathbf{x}_{\perp}, x_{j}=L, t\right)-V_{i}\left(\mathbf{x}_{\perp}, x_{j}=0, t\right)=0,
$$

where $\mathbf{x}_{\perp}$ are the coordinates orthogonal to $x_{j}$. Thus for the Fadeev-Popov determinant in Eq.(A5), we calculate

$$
\Delta_{f}[\mathbf{V}]=\left.\operatorname{det}\left(\frac{\partial f_{i}}{\partial c_{j}}\right)\right|_{\mathbf{c}=\mathbf{0}}=\operatorname{det}\left(-\delta_{i j}\right)=\int d \boldsymbol{\eta} d \boldsymbol{\eta}^{*} \exp \left(-i \boldsymbol{\eta}^{*} \cdot \boldsymbol{\eta}\right) .
$$

The last equality expresses this determinant as an integral over constant complex conjugate Grassmann vectors $\boldsymbol{\eta}$ and $\boldsymbol{\eta}^{*}$, with $\left\{\eta_{i}, \eta_{j}^{*}\right\}=0$ [10]. Lastly, since our gauge $\mathbf{f}(\mathbf{V})$ Eq.(7) is simply a constant vector, we repeat the arguments leading to Eq.(A6) and Eq.(A8) in the Appendix, but here replace the function $\mathbf{U}(\mathbf{x}, t) \rightarrow \mathbf{b}$ by a constant vector in Eq. (A7), and drop the spacetime integrations indicated there, that is, instead of Eq. (A7) we choose $G[\mathbf{b}]=\exp \left(-\frac{1}{2 \xi} \mathbf{b}^{2}\right)$, where $\xi>0$ is a free parameter.

Putting these results together, and after integrating over the delta -function in Eq.(A6), which in this case is $\delta\left(\mathbf{V}_{\mathbf{0}}-\mathbf{b}\right)$, the gauge fixed action reads:

$$
S_{G F}=S+\frac{1}{2 \xi} \mathbf{V}_{\mathbf{0}}^{2}+i \boldsymbol{\eta}^{*} \cdot \boldsymbol{\eta},
$$

where $S$ is given in Eq.(2) and we define $\mathbf{V}_{\mathbf{0}} \equiv \frac{1}{v o l} \int d \mathbf{x} d t \mathbf{V}(\mathbf{x}, t)$, and note that this has the correct dimensions of a velocity. Galilean invariance is manifestly broken in $S_{G F}$.

\section{B. Becchi-Rouet-Stora invariance of the gauge-fixed action}

Despite the fact that Galilean invariance has been broken explicitly, the gauge-fixed theory Eq.(12) does have a fundamental underlying symmetry which makes full use of the Grassmann vectors introduced to represent the Fadeev-Popov determinant Eq.(11). This symmetry was originally established to be a general consequence of gauge fixing in nonAbelian gauge theories, and is known as Becchi-Rouet-Stora (BRS) invariance ${ }^{3}$ [20].

\footnotetext{
${ }^{3}$ The BRS symmetry in our paper arises from gauge-fixing, and should thus be distinguished clearly from the BRS symmetry that arises from regarding the stochastic differential equation itself as the constraint; see, e.g., Ref. [8].
} 
Consider the following infinitesimal BRS transformation where $\zeta^{2}=0$ is a real Grassmann constant:

$$
\begin{aligned}
\delta_{\mathrm{BRS}} \mathbf{x} & =-\zeta\left(\boldsymbol{\eta}+\boldsymbol{\eta}^{*}\right) \\
\delta_{\mathrm{BRS}} t & =0 \\
\delta_{\mathrm{BRS}} \mathbf{V}(\mathbf{x}, t) & =\zeta\left(\eta_{k}+\eta_{k}^{*}\right) t \frac{\partial \mathbf{V}(\mathbf{x}, t)}{\partial x_{k}}-\zeta\left(\boldsymbol{\eta}+\boldsymbol{\eta}^{*}\right), \\
\delta_{\mathrm{BRS}} \boldsymbol{\sigma}(\mathbf{x}, t) & =\zeta\left(\eta_{k}+\eta_{k}^{*}\right) t \frac{\partial \boldsymbol{\sigma}(\mathbf{x}, t)}{\partial x_{k}}, \\
\delta_{\mathrm{BRS}} \boldsymbol{\eta} & =-\frac{i}{\xi} \mathbf{V}_{\mathbf{0}} \zeta \\
\delta_{\mathrm{BRS}} \boldsymbol{\eta}^{*} & =+\frac{i}{\xi} \mathbf{V}_{\mathbf{0}} \zeta .
\end{aligned}
$$

Note that Eq.(15) automatically implies the corresponding BRS transformation for the velocity zero mode $\mathbf{V}_{\mathbf{0}}$ :

$$
\delta_{B R S} \mathbf{V}_{\mathbf{0}}=\frac{1}{v o l} \int d \mathbf{x} d t \delta_{B R S} \mathbf{V}(\mathbf{x}, t)=-\zeta\left(\boldsymbol{\eta}+\boldsymbol{\eta}^{*}\right)
$$

Now Eqs.(13,[16) are recognized to be just the infinitesimal version of the Galilean transformation Eqs.(3-6), but with the boost velocity replaced by the Grassmann vectors $\mathbf{c} \rightarrow \zeta\left(\boldsymbol{\eta}+\boldsymbol{\eta}^{*}\right)$. Therefore, the action $S$ and path integral measure are automatically invariant under this subset of the full BRS transformation. Next, from Eqs.(17],18]19), we easily verify that the combined gauge fixing and Grassmann terms in $S_{G F}$ are invariant. From these observations we therefore conclude that the complete gauge fixed action $S_{G F}$ in Eq.(12) is invariant under the full BRS transformation Eqs.(13), 18):

$$
\delta_{B R S} S_{G F}=0
$$

This BRS invariance leads to exact identities which we derive below.

\section{The Slavnov-Taylor and Ward identities}

We introduce source terms into the gauge-fixed functional as follows:

$$
\begin{aligned}
Z_{G F}\left[\mathbf{J}, \boldsymbol{\Sigma}, \boldsymbol{\theta}, \boldsymbol{\theta}^{*}\right] & =\int[D \mathbf{V}][D \boldsymbol{\sigma}] d \boldsymbol{\eta} d \boldsymbol{\eta}^{*} \exp \left\{-S_{G F}\left[\mathbf{V}, \boldsymbol{\sigma}, \boldsymbol{\eta}, \boldsymbol{\eta}^{*}\right]+\boldsymbol{\theta}^{*} \cdot \boldsymbol{\eta}+\boldsymbol{\theta} \cdot \boldsymbol{\eta}^{*}\right. \\
& \left.+\int d \mathbf{x} d t\{\mathbf{J}(\mathbf{x}, t) \cdot \mathbf{V}(\mathbf{x}, t)+\boldsymbol{\Sigma}(\mathbf{x}, t) \cdot \boldsymbol{\sigma}(\mathbf{x}, t)\}\right\},
\end{aligned}
$$


where $\boldsymbol{\theta}$ and $\boldsymbol{\theta}^{*}$ are complex Grassmann vectors. Next, we subject this functional to the complete BRS transformation (that is, we displace all fields by the infinitesimal BRS transformation) Eqs.(13,-18). Since the measure and the gauge-fixed action $S_{G F}$ are BRS-invariant, only the source terms in Eq.(21) will be affected. Moreover, as $\zeta^{2}=0$, we can easily expand the exponential: $\exp (\zeta A)=1+\zeta A$. The gauge-fixed functional Eq.(21) thus transforms as $Z_{G F} \rightarrow Z_{G F}+\delta_{B R S} Z_{G F}$, where $\delta_{B R S} Z_{G F}=0$ can be written as follows:

$$
\left[\frac{i}{\xi}\left(\boldsymbol{\theta}^{*}-\boldsymbol{\theta}\right) \cdot \frac{1}{v o l} \int d \mathbf{x} d t \frac{\delta}{\delta \mathbf{J}(\mathbf{x}, t)}+\left(\frac{\partial}{\partial \theta_{j}}+\frac{\partial}{\partial \theta_{j}^{*}}\right) \hat{O}_{j}\right] Z_{G F}\left[\mathbf{J}, \boldsymbol{\Sigma}, \boldsymbol{\theta}, \boldsymbol{\theta}^{*}\right]=0 .
$$

Here the operator

$$
\hat{O}_{j}=\int d \mathbf{x} d t\left(J_{m} t \nabla_{j} \frac{\delta}{\delta J_{m}}+\Sigma_{m} t \nabla_{j} \frac{\delta}{\delta \Sigma_{m}}-J_{j}\right)
$$

is the same operator appearing in the well known Ward identity that follows from the Galilean invariance of the non-gauge fixed functional $\left[2,\left[3,4\right.\right.$, 6]: that is, $\hat{O}_{j} Z[\boldsymbol{J}, \boldsymbol{\Sigma}]=0$.

To finish, we introduce the effective action $\Gamma$ and write the Slavnov-Taylor identity Eq.(22) in terms of this quantity. Let $W=\ln Z_{G F}$, then the generating functional of one-particle irreducible functions is given by the Legendre transform

$$
\Gamma\left[\mathbf{V}_{c l}, \boldsymbol{\sigma}_{c l}, \boldsymbol{\eta}_{c l}, \boldsymbol{\eta}_{c l}^{*}\right]=-W\left[\mathbf{J}, \boldsymbol{\Sigma}, \boldsymbol{\theta}, \boldsymbol{\theta}^{*}\right]+\boldsymbol{\theta}^{*} \cdot \boldsymbol{\eta}_{c l}+\boldsymbol{\theta} \cdot \boldsymbol{\eta}_{c l}^{*}+\int d \mathbf{k} d \omega\left(\mathbf{J} \cdot \mathbf{V}_{c l}+\boldsymbol{\Sigma} \cdot \boldsymbol{\sigma}_{c l}\right)
$$

which for convenience, we express in wavevector $\mathbf{k}$ and frequency space $\omega$. Here, the label " $c l$ " reminds us that the fields so indicated are averaged over the fluctuating fields and in the presence of the source terms: that is, using the ensemble in Eq.(21). From Eq.(24) we have that

$$
J_{k}=\frac{\delta \Gamma}{\delta V_{k}^{c l}}, \Sigma_{k}=\frac{\delta \Gamma}{\delta \sigma_{k}^{c l}}, \theta_{j}=\frac{\partial \Gamma}{\partial \eta_{j}^{* c l}}, \theta_{j}^{*}=\frac{\partial \Gamma}{\partial \eta_{j}^{c l}} .
$$

We can now straightforwardly write down the identity for $\Gamma$ that follows directly from $\delta_{B R S} Z_{G F}=0$ :

$$
\begin{aligned}
& 0=\frac{i}{\xi} \mathbf{V}_{0}^{c l} \cdot\left(\frac{\partial \Gamma}{\partial \boldsymbol{\eta}_{c l}}-\frac{\partial \Gamma}{\partial \boldsymbol{\eta}_{c l}^{*}}\right)+\left(\eta_{c l}+\eta_{c l}^{*}\right)_{j} \times \\
& \int d \mathbf{k} d \omega\left(k_{j} \frac{\partial V_{m}^{c l}(\mathbf{k}, \omega)}{\partial \omega} \frac{\delta \Gamma}{\delta V_{m}^{c l}(\mathbf{k}, \omega)}+k_{j} \frac{\partial \sigma_{m}^{c l}(\mathbf{k}, \omega)}{\partial \omega} \frac{\delta \Gamma}{\delta \sigma_{m}^{c l}(\mathbf{k}, \omega)}-\delta(\mathbf{k}) \delta(\omega) \frac{\delta \Gamma}{\delta V_{j}^{c l}(\mathbf{k}, \omega)}\right) .
\end{aligned}
$$

We now make use of this formula and apply it to the problem at hand. The dependence of $\Gamma$ on $\boldsymbol{\eta}$ and $\boldsymbol{\eta}^{*}$ is simple since these constant Grassmann vector fields do not interact nor do 
they couple to the velocity or conjugate fields ${ }^{4}$. Thus, we can immediately write

$$
\Gamma\left[\mathbf{V}_{c l}, \boldsymbol{\sigma}_{c l}, \boldsymbol{\eta}_{c l}, \boldsymbol{\eta}_{c l}^{*}\right]=-i \boldsymbol{\eta}_{c l}^{*} \cdot \boldsymbol{\eta}_{c l}+\Gamma\left[\mathbf{V}_{c l}, \boldsymbol{\sigma}_{c l}\right]
$$

where $\Gamma\left[\mathbf{V}_{c l}, \sigma_{c l}\right]$ does not depend on either $\boldsymbol{\eta}_{c l}$ or $\boldsymbol{\eta}_{c l}^{*}$, and the first few required terms are written out in Eq. (B1) in Appendix B.

The pertinent identity we seek is then obtained by inserting Eq.(27) into Eq.(26), and then differentiating this with respect to $\delta / \delta V_{l}^{c l}(\mathbf{k}, \omega) \delta / \delta \sigma_{n}^{c l}(-\mathbf{k},-\omega)$, and then setting $\mathbf{V}^{c l}$ $=\boldsymbol{\sigma}^{c l}=0$. Note that the terms in Eq.(26) depending on the gauge parameter $\xi$ do not contribute to this sequence of steps, and most importantly, we end up obtaining the following result:

$$
\left(\eta_{c l}+\eta_{c l}^{*}\right)_{j}\left(k_{m} \frac{\partial}{\partial \omega} \Gamma_{l n}^{(1,1)}(\mathbf{k}, \omega ;-\mathbf{k},-\omega)+\Gamma_{m l n}^{(2,1)}(\mathbf{0}, 0 ; \mathbf{k}, \omega ;-\mathbf{k},-\omega)\right)=0
$$

Moreover, since $\boldsymbol{\eta}_{c l}$ and $\boldsymbol{\eta}_{c l}^{*}$ are arbitrary, the expression within the larger parentheses must vanish identically. $\Gamma^{(1,1)}$ and $\Gamma^{(2,1)}$ denote the inverse response and vertex functions, respectively. Thus, we recover the well-known Ward identity derived previously on many occasions from the non-gauge fixed action [3, 4, 6, 7]. Alternatively, we can differentiate Eq.(26) with respect to $\partial / \partial \eta_{j}^{c l} \delta / \delta V_{l}^{c l}(\mathbf{k}, \omega) \delta / \delta \sigma_{n}^{c l}(-\mathbf{k},-\omega)$ followed by setting $\boldsymbol{\eta}^{c l}=\boldsymbol{\eta}_{c l}^{*}=\mathbf{V}^{c l}=\boldsymbol{\sigma}^{c l}=\mathbf{0}$. The end result is the same.

In summary, the Ward identity Eq.(28) relating the exact inverse response function to the exact vertex of the NSE holds for the gauge fixed action Eq.(12), is a direct consequence of the BRS invariance of the latter, and is independent of the gauge parameter $\xi$. From this point on, the entire discussion regarding the implications of Galilean invariance for the vertex renormalization [7] continues to apply; see Sec V]. Most importantly, we see that those arguments are valid even after breaking the Galilean invariance and going to a fixed reference frame.

\section{The $\delta$-gauge}

Other gauge choices are possible. To briefly illustrate this, we consider this field theory in the $\delta$-gauge, in which we work directly with the functional integral in the form Eq. (A66)

\footnotetext{
4 This dependence of $\Gamma$ on the Grassmann variables is similar to that for gauge-fixed quantum electrodynamics, see, e.g. the development in Ramond's book [10].
} 
(and with the overall infinite term removed). Put $\mathbf{f}(\mathbf{V})=\mathbf{V}_{\mathbf{0}}$ and $\mathbf{U}=\mathbf{b}$ in Eq.(A6), choose as the weight function $G[\mathbf{U}]=1$ rather than Eq.(A7), and use the representation

$$
\delta^{3}\left(\mathbf{V}_{0}-\mathbf{b}\right)=\int \exp \left[i \mathbf{K} \cdot\left(\mathbf{V}_{0}-\mathbf{b}\right)\right] \frac{d^{3} K}{(2 \pi)^{3}}
$$

In this case, by using the final equality in Eq.(11), for expressing the determinant of the identity matrix as a Grassmann integral, it gives

$$
S_{G F}=S[\mathbf{V}, \boldsymbol{\sigma}]-i \mathbf{K} \cdot\left(\mathbf{V}_{0}-\mathbf{b}\right)+i \boldsymbol{\eta}^{*} \cdot \boldsymbol{\eta}
$$

The Galilean transformations are as above Eqs.(3] - 6), for which $S[\mathbf{V}, \boldsymbol{\sigma}]$ and the measure are invariant. For the BRS transformation, we identify $\mathbf{c} \rightarrow \zeta\left(\boldsymbol{\eta}^{*}+\boldsymbol{\eta}\right)$ as before with

$$
\begin{aligned}
\delta_{\mathrm{BRS}} \mathbf{V}_{0 j} & =-\zeta\left(\eta_{j}^{*}+\eta_{j}\right), \\
\delta_{\mathrm{BRS}} \eta_{j} & =\mathbf{K}_{j} \zeta \\
\delta_{\mathrm{BRS}} \eta_{j}^{*} & =-\mathbf{K}_{j} \zeta, \\
\delta_{\mathrm{BRS}} \mathbf{b} & =\delta_{\mathrm{BRS}} \mathbf{K}=0 .
\end{aligned}
$$

We point out that $\boldsymbol{\eta}, \boldsymbol{\eta}^{*}$ need not be a complex conjugate pair; see footnote in Sec IIIC. With this, one can check

$$
\delta_{B R S}\left(i \boldsymbol{\eta}^{*} \cdot \boldsymbol{\eta}-i \mathbf{K} \cdot\left(\mathbf{V}_{0}-\mathbf{b}\right)\right)=0
$$

proving that the gauge-fixed action in this gauge Eq.(30) is BRS invariant: $\delta_{B R S} S_{G F}=0$.

\section{E. Ward identity in a box and spurious relations}

In order to remove any ambiguity from our treatment of the continuum NSE path integral in [13] and the spurious relations we found there, we here perform a similar analysis but now for a discrete and hence regularized version of NSE field theory. In this Section we use the dynamic functional $Z$ for the NSE derived in a box (see Appendix C) to obtain the associated Ward identities. An important technical distinction stems from the fact that in a box, there is a smallest limiting finite velocity boost. Only in the continuum can infinitesimal boosts be treated. 
To begin, and including the sources from the outset, we start with

$$
\begin{aligned}
Z[\mathbf{J}, \boldsymbol{\Sigma}]= & \int \prod_{\alpha, \mathbf{n}, j} d v_{\alpha}(\mathbf{n}, j) d \sigma_{\alpha}(\mathbf{n}, j) \times \\
& \exp \left(-S[\mathbf{v}, \boldsymbol{\sigma}]+\sum_{\mathbf{m}, l}\left(J_{\alpha}(-\mathbf{m},-l) v_{\alpha}(\mathbf{m}, l)+\Sigma_{\alpha}(-\mathbf{m},-l) \sigma_{\alpha}(\mathbf{m}, l)\right)\right) .
\end{aligned}
$$

Here, the discrete action $S$ is given by Eq. (C9). We repeat all the steps that for the continuum case, lead one to obtain the Ward as for example in [7]. Here, we carry this out for the discrete theory. So we next transform $Z$ using the discrete version of the Galilean transformation Eq.(C12). Doing so we obtain:

$$
\begin{aligned}
Z[\mathbf{J}, \boldsymbol{\Sigma}] & =\int \prod_{\alpha, \mathbf{n}, j} d v_{\alpha}(\mathbf{n}, j) d \sigma_{\alpha}(\mathbf{n}, j) \exp \left(-S[\mathbf{v}, \boldsymbol{\sigma}]+\sum_{\mathbf{m}, l}\left(J_{\alpha}(-\mathbf{m},-l) v_{\alpha}(\mathbf{m}, l+\overline{\mathbf{c}} \cdot \mathbf{m})\right.\right. \\
& \left.\left.-\overline{\mathbf{c}} \cdot \mathbf{J}(-\mathbf{m},-l) \delta^{3}(\mathbf{m}) \delta(l)+\Sigma_{\alpha}(-\mathbf{m},-l) \sigma_{\alpha}(\mathbf{m}, j+\overline{\mathbf{c}} \cdot \mathbf{m})\right)\right) .
\end{aligned}
$$

Now unlike the continuum case, we cannot consider an infinitesimal boost velocity. Instead, the best we can do is consider the smallest nonzero boost velocity, which, according to comments immediately below Eq.(C12), is $\delta \bar{c}=(1,1,1)$. We could as well have chosen $(1,0,0)$, or $(0,1,0)$, or $(0,0,1)$, or $(1,1,0)$, etc. The point is, the smallest allowable boost velocity is not infinitesimal.

We next emulate what is done for the continuum case, namely Taylor expand the velocity and conjugate fields about zero boost, see for example, Eq.(4) in [7]. The best we can do is to employ the following approximation:

$$
\begin{aligned}
v_{\alpha}(\mathbf{m}, l+\overline{\mathbf{c}} \cdot \mathbf{m}) & =v_{\alpha}(\mathbf{m}, l)+\overline{\mathbf{c}} \cdot \mathbf{m} \Delta v_{\alpha}(\mathbf{m}, l) \\
& =v_{\alpha}(\mathbf{m}, l)+\left(m_{1}+m_{2}+m_{3}\right) \Delta v_{\alpha}(\mathbf{m}, l)
\end{aligned}
$$

where the finite difference operator $\Delta f(\mathbf{m}, l)=f(\mathbf{m}, l+1)-f(\mathbf{m}, l)$.

To finish the derivation, we insert these finite difference approximations back into Eq.(37). This implies that the functional transforms as $Z \rightarrow Z+\delta Z$. We require that the extra terms proportional to the boost velocity $\overline{\mathbf{c}}$ vanish identically. This leads to:

$$
\begin{aligned}
\sum_{\mathbf{m}, l} & \left(J_{\alpha}(-\mathbf{m},-l) m_{i} \Delta \frac{\partial}{\partial J_{\alpha}(-\mathbf{m},-l)}\right. \\
& \left.+\Sigma_{\alpha}(-\mathbf{m},-l) m_{i} \Delta \frac{\partial}{\partial \Sigma_{\alpha}(-\mathbf{m},-l)}-J_{i}(-\mathbf{m},-l) \delta_{\mathbf{m}, \mathbf{0}}^{3} \delta_{l, 0}\right) Z[\mathbf{J}, \mathbf{\Sigma}]=0 .
\end{aligned}
$$


This is the Ward identity for $Z$ and should be compared to the Fourier transform of the continuum version Eq.(6) in [7].

Differentiate this WTI for $Z$, Eq.(139), with respect to $\left.\frac{\partial}{\partial J(-\mathbf{m},-l)}\right|_{J=\Sigma=0}$, to obtain

$$
m_{i} \Delta<v_{k}(\mathbf{m}, l)>-\delta_{i k}=0 .
$$

This is well behaved and never singular, but it suffers from the same defect as its continuum version [13] when we set $i=k$ and sum over this index: fluid incompressibility implies $m_{i} v_{i}(\mathbf{m}, l)=0$, which since it is zero, leads to a contradiction in Eq.(40).

For higher point correlations, more spurious relations are found from Eq.(39), similar to those found in the continuum case in [13]. The source of these spurious relations is the same as in [13], and we can verify that the functional integral in Eq.(37) has an infinite prefactor due to the integration over the velocity zero mode. This can be fixed by the same gauge fixing procedure as we carried out in [13], taking appropriate modifications for the discrete case. Most importantly, the treatment in this Section demonstrates that these spurious relations are not an artifact of the continuum path integral expressions.

\section{EXTENDED GALILEAN INVARIANCE (EGI): GAUGE FIXING AND BRS SYMMETRY}

Galilean invariance admits an interesting physical extension. As it turns out, the NavierStokes (NSE) equation is invariant under rectilinear frame accelerations, a symmetry known as extended Galilean invariance (EGI) [21]. This invariance was noted and used in the context of the KPZ equation [22], as well as in the stochastic Burgers equation [23], it has been used in probability density functions in turbulence modeling [24], and finds practical applications in fluid animation simulations [25]. We review this invariance of the NSE below. We then proceed to gauge-fix this symmetry, write down the corresponding BRS transformation that leaves the gauge-fixed action invariant, and then deduce the associated Slavnov-Taylor identity for the functional and the new generalized Ward identity that follows from it. 


\section{A. Extended Galilean invariance of the Navier-Stokes equation}

Consider the Navier-Stokes equation (NSE) in an inertial frame $E$ :

$$
\frac{\partial V_{i}}{\partial t}+\frac{\partial\left(V_{i} V_{j}\right)}{\partial x_{j}}=-\frac{\partial \Pi}{\partial x_{i}}+\nu \nabla^{2} V_{i}
$$

where $\nu$ is the kinematic viscosity of the fluid, $V_{i}(\mathbf{x}, t)$ and $\Pi(\mathbf{x}, t)$ are the instantaneous values of the velocity and pressure, and the continuity equation takes the form

$$
\frac{\partial V_{i}}{\partial x_{i}}=0
$$

for an incompressible fluid. In this case, the density is constant for all $\mathbf{x}$ and $t$ so for convenience we may work in a system of units where the fluid density is taken to be unity. Also, as is well known, taking the divergence of each term in Eq.(41), and invoking Eq.(42), leads to a Poisson-type equation for the pressure, viz.,

$$
\nabla^{2} \Pi=-\frac{\partial^{2}\left(V_{i} V_{j}\right)}{\partial x_{i} \partial x_{j}}
$$

and this result is useful for establishing the Galilean transformation of the fluid pressure.

Now consider a second noninertial frame $\bar{E}$ moving with respect to $E$ with a variable but rectilinear velocity $\mathbf{c}(t)$. The transformation between these two coordinate systems is as follows,

$$
\begin{aligned}
\mathbf{x}^{\prime} & =\mathbf{x}-\boldsymbol{\lambda}(t), \\
t^{\prime} & =t, \\
\mathbf{V}^{\prime}\left(\mathbf{x}^{\prime}, t^{\prime}\right) & =\mathbf{V}(\mathbf{x}, t)-\dot{\lambda}(t), \\
\Pi^{\prime}\left(\mathbf{x}^{\prime}, t^{\prime}\right) & =\Pi(\mathbf{x}, t)+\mathbf{x}^{\prime} \cdot \ddot{\lambda}(t),
\end{aligned}
$$

where $\boldsymbol{\lambda}(t)=\int_{0}^{t} \mathbf{c}(s) d s$, and the dots stand for the derivative taken with respect to time. Applying these transformation rules to (41) proves that the transformed NSE takes the form

$$
\frac{\partial V_{i}^{\prime}}{\partial t^{\prime}}+\frac{\partial\left(V_{i}^{\prime} V_{j}^{\prime}\right)}{\partial x_{j}^{\prime}}=-\frac{\partial \Pi^{\prime}}{\partial x_{i}^{\prime}}+\nu \nabla^{\prime 2} V_{i}^{\prime},
$$

which is the NSE in the noninertial accelerating frame. Note that $\ddot{\boldsymbol{\lambda}}(t)=\dot{\mathbf{c}}(t)=\mathbf{A}(t)$, so that the frame acceleration is absorbed into a modified pressure Eq.(47). This demonstrates 
that the NSE Eq.(41) is invariant under rectilinear frame accelerations. It is clear that when the above transformation rules Eqs.(44-47) include one for the conjugate field:

$$
\boldsymbol{\sigma}^{\prime}\left(\mathbf{x}^{\prime}, t^{\prime}\right)=\boldsymbol{\sigma}(\mathbf{x}, t)
$$

then the action Eq.(2) and dynamic functional Eq.(1) are invariant as well under this extended Galilean transformation.

\section{B. Gauge Fixing for Extended Galilean invariance}

We next gauge-fix the EGI of the dynamic functional in Eq.(1). Once again, we refer to the general gauge-fixing procedure as outlined in Appendix A. We make the specific gauge choice introduced below, calculate the corresponding Fadeev-Popov determinant Eq.(A5), and once again select the arbitrary function $\mathbf{U}$ that appears in Eq. (A6) to complete the procedure.

In the case of EGI, the purpose of gauge fixing is to single out one rectilinearly accelerating reference frame. This can be accomplished by now constraining the time-dependent zero mode of the full instantaneous velocity field. This mode corresponds to the acceleration of the system as a whole, that is, its bulk acceleration. We therefore make the following choice for the gauge function, where $v o l=L^{3}$ is the volume of the spatially bounded box:

$$
\mathbf{f}(\mathbf{V})=\frac{1}{v o l} \int d \mathbf{x} \mathbf{V}(\mathbf{x}, t) \equiv \mathbf{V}_{\mathbf{0}}(t)
$$

This projects out the instantaneous and arbitrary time dependent bulk velocity of the entire bounded system as required. From Eqs.(44,46) we have

$$
\begin{aligned}
V_{j}^{\boldsymbol{\lambda}}(\mathbf{x}, t) & =V_{j}^{\prime}\left(\mathbf{x}^{\prime}, t^{\prime}\right)+\dot{\lambda}_{j}(t), \\
& =V_{j}^{\prime}(\mathbf{x}-\boldsymbol{\lambda}(t), t)+\dot{\lambda}_{j}(t), \\
& =V_{j}^{\prime}(\mathbf{x}, t)-\lambda_{k}(t) \frac{\partial}{\partial x_{k}} V_{j}^{\prime}(\mathbf{x}, t)+\dot{\lambda}_{j}(t)+O\left(\lambda^{2}\right), \\
\text { So }\left.\frac{\delta V_{j}^{\boldsymbol{\lambda}}(\mathbf{x}, t)}{\delta \lambda_{m}\left(t^{\prime}\right)}\right|_{\boldsymbol{\lambda}=\mathbf{0}} & =\left(\frac{\partial V_{j}^{\prime}(\mathbf{x}, t)}{\partial x_{m}}-\delta_{j m} \frac{d}{d t}\right) \delta\left(t-t^{\prime}\right) .
\end{aligned}
$$

The FP determinant Eq. An this case works out to be, again using periodic boundary conditions for the bounded spatial box,

$$
\begin{aligned}
\Delta_{f}[\mathbf{V}]=\left.\operatorname{det}\left(\frac{\delta f_{i}\left(t ; \mathbf{V}^{\lambda}\right)}{\delta \lambda_{m}\left(t^{\prime}\right)}\right)\right|_{\boldsymbol{\lambda}=\mathbf{0}} & =\operatorname{det}\left(-\delta_{i m} \frac{d}{d t} \delta\left(t-t^{\prime}\right)\right) \\
& =\int \mathcal{D} \boldsymbol{\eta} \mathcal{D} \boldsymbol{\eta}^{*} \exp \left(-i \int d t \boldsymbol{\eta}(t)^{*} \cdot \frac{d}{d t} \boldsymbol{\eta}(t)\right)
\end{aligned}
$$


The functional integral in Eq.(55) is over two time-dependent Grassman vector fields $\boldsymbol{\eta}(t)$ and $\boldsymbol{\eta}^{*}(t)$. For the convergent integral over the gauge group volume, we now choose (see Eq. (A7) in Appendix A)

$$
G[\mathbf{b}]=\exp \left(-\frac{1}{2 \xi} \int d t \mathbf{b}^{2}(t)\right)
$$

which yields the gauge-fixed action for EGI:

$$
S_{G F}=S[\mathbf{V}, \boldsymbol{\sigma}]+\int d t\left\{\frac{1}{2 \xi} \mathbf{V}_{\mathbf{0}}^{2}(t)+i \boldsymbol{\eta}^{*}(t) \frac{d}{d t} \boldsymbol{\eta}(t)\right\}
$$

At this point, Extended Galilean invariance is manifestly broken in $S_{G F}$.

\section{Becchi-Rouet-Stora transformation for gauge fixed EGI}

We begin by writing down the BRS transformation. We observe that the transformations in Eqs. (58,62) below are just the infinitesimal version of the extended Galilean transformations Eqs.(44,47), but with the variable rectilinear boost velocity now replaced by $\dot{\boldsymbol{\lambda}}(t)=\mathbf{c}(t) \rightarrow \zeta \dot{\boldsymbol{\eta}}(t)$, where $\zeta$ is a real constant Grassmann parameter: $\zeta^{2}=0$. Therefore the nongauge-fixed action $S$ and the path integral measure are automatically invariant under this subset of BRS transformations:

$$
\begin{aligned}
\delta_{\mathrm{BRS}} \mathbf{x} & =-\zeta \boldsymbol{\eta}(t) \\
\delta_{\mathrm{BRS}} t & =0 \\
\delta_{\mathrm{BRS}} \mathbf{V}(\mathbf{x}, t) & =\zeta \eta_{k}(t) \frac{\partial \mathbf{V}(\mathbf{x}, t)}{\partial x_{k}}-\zeta \dot{\boldsymbol{\eta}}(t), \\
\delta_{\mathrm{BRS}} \boldsymbol{\sigma}(\mathbf{x}, t) & =\zeta \eta_{k}(t) \frac{\partial \boldsymbol{\sigma}(\mathbf{x}, t)}{\partial x_{k}} \\
\delta_{\mathrm{BRS}} \Pi(\mathbf{x}, t) & =\zeta \eta_{k}(t) \frac{\partial \Pi(\mathbf{x}, t)}{\partial x_{k}}+\mathbf{x} \cdot \zeta \ddot{\boldsymbol{\eta}}(t) .
\end{aligned}
$$

To complete the BRS transformation, we need to know how the velocity zero mode $\mathbf{V}_{\mathbf{0}}(t)$ and Grassmann sector transform. The remaining BRS transformations are given as follows:

$$
\begin{aligned}
\delta_{\mathrm{BRS}} \mathbf{V}_{\mathbf{0}}(t) & =-\zeta \dot{\boldsymbol{\eta}}(t) \\
\delta_{\mathrm{BRS}} \boldsymbol{\eta}(t) & =0 \\
\delta_{\mathrm{BRS}} \boldsymbol{\eta}^{*}(t) & =\frac{-i}{\xi} \mathbf{V}_{\mathbf{0}}(t) \zeta
\end{aligned}
$$


Note that Eq.(63) is an immediate consequence of Eq.(60). Secondly, note the rather different ways that $\boldsymbol{\eta}$ and $\boldsymbol{\eta}^{*}$ transform. This "asymmetry" is in fact familiar from non-abelian quantum field theory ${ }^{5}$

Summarizing up to this point, we have demonstrated that the gauge fixed action Eq.(57) is invariant under the above BRS transformation:

$$
\delta_{\mathrm{BRS}} S_{G F}=0 .
$$

\section{The Generalized Slavnov-Taylor and Ward identities}

To conclude, we subject $Z_{G F}$, which we now define below, to the BRS transformation and derive the generalized Ward identity that holds for $\Gamma$,

$$
\begin{aligned}
Z_{G F}\left[\mathbf{J}, \boldsymbol{\Sigma}, \boldsymbol{\theta}, \boldsymbol{\theta}^{*}\right] & =\int[D \mathbf{V}][D \boldsymbol{\sigma}][D \boldsymbol{\eta}]\left[D \boldsymbol{\eta}^{*}\right] \exp \left\{-S_{G F}\left[\mathbf{V}, \boldsymbol{\sigma}, \boldsymbol{\eta}, \boldsymbol{\eta}^{*}\right]+\int d t\left(\boldsymbol{\theta}^{*} \cdot \boldsymbol{\eta}+\boldsymbol{\theta} \cdot \boldsymbol{\eta}^{*}\right)\right. \\
& \left.+\int d \mathbf{x} d t\{\mathbf{J}(\mathbf{x}, t) \cdot \mathbf{V}(\mathbf{x}, t)+\boldsymbol{\Sigma}(\mathbf{x}, t) \cdot \boldsymbol{\sigma}(\mathbf{x}, t)\}\right\}
\end{aligned}
$$

Here of course, the gauge fixed action $S_{G F}$ is given by Eq.(57), and the Grassmann vector sources $\boldsymbol{\theta}(t), \boldsymbol{\theta}^{*}(t)$ are now time dependent. We next subject this functional to the complete BRS transformation listed in Eqs.(58,65). Just as in the case treated in Sec [IC, the measure and gauge-fixed action are invariant and only the source terms will be affected. The remainder of the steps involved are similar to those employed above. We expand the exponential to first order in $\zeta$, and then make use of the Legendre transform similar to Eq.(24) to define the effective action $\Gamma$. For the present case, the Grassmann source terms are integrated over time in the Legendre transform. After a few simple steps we arrive at the following Slavnov-Taylor identity:

$$
\left[\frac{i}{\xi} \int d t \frac{\delta \Gamma}{\delta \boldsymbol{\eta}_{c l}^{*}(t)} \cdot \mathbf{V}_{\mathbf{0}}^{c l}(t)+\int d \mathbf{x} \int d t \eta_{j}^{c l}(t)\left(\frac{\delta \Gamma}{\delta V_{l}^{c l}} \nabla_{j} V_{l}^{c l}+\frac{\delta \Gamma}{\delta \sigma_{l}^{c l}} \nabla_{j} \sigma_{l}^{c l}+\frac{\partial}{\partial t} \frac{\delta \Gamma}{\delta V_{j}^{c l}}\right)\right]=0 .
$$

\footnotetext{
5 See for example the chapter on "Yang-Mills Theory: Slavnov-Taylor Identities", page 370 in [10] for full details of the proof of the BRS invariance of gauge-fixing and ghost terms in a general Yang-Mills gauge theory. Note also the use there of two independent real Grassmann fields. The limit of vanishing Lie algebra structure constants (the Abelian limit) in Yang-Mills quantum field theory implies that one of the two ghost fields transforms to zero under BRS. See also remarks [26] to the effect that $\boldsymbol{\eta}$ and $\boldsymbol{\eta}^{*}$ can be independent, it is not necessary to think of them as hermitian conjugates, indeed they can be any two independent anticommuting fields.
} 
The pertinent Ward identity we seek is obtained by inserting the functional Taylor series for $\Gamma$ (see e.g., Eq.(9,10) of Ref.[7]) into Eq.([68), differentiating the latter with respect to $V_{k}\left(\mathbf{y}, t^{\prime}\right)$ and $\sigma\left(\mathbf{w}, t^{\prime \prime}\right)$, and then setting all the fields equal to zero. We immediately see that term depending on the gauge parameter $\xi$ will not survive this sequence of steps and furthermore, since $\boldsymbol{\eta}^{c l}(t)$ is an arbitrary function of time, the identity Eq. (68) effectively reduces to

$$
\int d^{d} \mathbf{x}\left(\nabla_{j} V_{l}(\mathbf{x}, t) \frac{\delta \Gamma}{\delta V_{l}(\mathbf{x}, t)}+\nabla_{j} \sigma_{l}(\mathbf{x}, t) \frac{\delta \Gamma}{\delta \sigma_{l}(\mathbf{x}, t)}+\frac{\partial}{\partial t} \frac{\delta \Gamma}{\delta V_{j}(\mathbf{x}, t)}\right)=0 .
$$

Carrying out the specific differentiations mentioned above yields a relation between the exact inverse response $\Gamma^{(1,1)}$ and the vertex functions $\Gamma^{(2,1)}$ :

$$
\begin{aligned}
\frac{\partial}{\partial t} \int d^{d} \mathbf{x} \Gamma_{n k j}^{(2,1)}\left(\mathbf{x}, t ; \mathbf{y}, t^{\prime} ; \mathbf{w}, t^{\prime \prime}\right) & =\delta\left(t-t^{\prime \prime}\right) \frac{\partial}{\partial w_{n}} \Gamma_{k j}^{(1,1)}\left(\mathbf{y}, t^{\prime} ; \mathbf{w}, t\right) \\
& +\delta\left(t-t^{\prime}\right) \frac{\partial}{\partial y_{n}} \Gamma_{k j}^{(1,1)}\left(\mathbf{y}, t ; \mathbf{w}, t^{\prime \prime}\right)
\end{aligned}
$$

This is the new Ward identity that follows from EGI in configuration space and time, derived here for the first time. Whereas the vertex function in the conventional Ward identity (see, e.g., Eq.(11) of Ref.[7]) is integrated over both coordinates and time, here by contrast, the vertex is integrated only over coordinates and is differentiated with respect to time. Using translational invariance (in space and in time), Fourier transforming (170) and then integrating over $\int d t \int d t^{\prime} \int d t^{\prime \prime} e^{-i \omega_{1} t} e^{-i \omega_{2} t^{\prime}} e^{-i \omega_{3} t^{\prime \prime}}$ to get rid of the delta-functions, we obtain the Ward identity in wavevector and frequency space (note: here, $\nu$ stands for a frequency, not the viscosity):

$$
k_{n} \Gamma_{k j}^{(1,1)}(\mathbf{k}, \omega+\nu ;-\mathbf{k},-\omega-\nu)-k_{n} \Gamma_{k j}^{(1,1)}(\mathbf{k}, \nu ;-\mathbf{k},-\nu)=-\omega \Gamma_{n k j}^{(2,1)}(\mathbf{0}, \omega ; \mathbf{k}, \nu ;-\mathbf{k}, \omega-\nu) .
$$

If we take the zero frequency limit $\omega \rightarrow 0$, we then obtain the identity

$$
k_{n} \frac{\partial}{\partial \nu} \Gamma_{k j}^{(1,1)}(\mathbf{k}, \nu ;-\mathbf{k},-\nu)=-\Gamma_{n k j}^{(2,1)}(\mathbf{0}, 0 ; \mathbf{k}, \nu ;-\mathbf{k},-\nu),
$$

which is precisely the Ward identity Eq.(28) that follows from the standard Galilean invariance of the NSE, that is, when the frame $\bar{E}$ moves with a constant velocity $\ddot{\boldsymbol{\lambda}}(t)=\mathbf{0}$ with respect to the lab frame $E[7]$.

For EGI, there is second way to implement the BRS transformation, intimately related to how we choose to define the gauge-function. Thus, if we now choose $\dot{\boldsymbol{\lambda}}(t)$ instead of $\boldsymbol{\lambda}(t)$ 
as the gauge function, then in place of Eq.(54), we would have, after using the identity $\lambda_{k}(t)=\int_{0}^{t} d u \frac{d \lambda_{k}(u)}{d u}=\int_{0}^{t} d u \dot{\lambda}_{k}(u)$, the relation

$$
\left.\frac{\delta V_{j}^{\boldsymbol{\lambda}}(\mathbf{x}, t)}{\delta \dot{\lambda}_{m}\left(t^{\prime}\right)}\right|_{\dot{\boldsymbol{\lambda}}=\mathbf{0}}=\frac{\partial V_{j}^{\prime}(\mathbf{x}, t)}{\partial x_{m}}-\delta_{j m} \delta\left(t-t^{\prime}\right) .
$$

The FP determinant, invoking the same boundary conditions as before, is now given by

$$
\begin{aligned}
\Delta_{f}[\mathbf{V}]=\left.\operatorname{det}\left(\frac{\delta f_{i}\left(t ; \mathbf{V}^{\lambda}\right)}{\delta \dot{\lambda}_{m}\left(t^{\prime}\right)}\right)\right|_{\dot{\lambda}=\mathbf{0}} & =\operatorname{det}\left(-\delta_{i m} \delta\left(t-t^{\prime}\right)\right) \\
& =\int \mathcal{D} \boldsymbol{\eta} \mathcal{D} \boldsymbol{\eta}^{*} \exp \left(-i \int d t \boldsymbol{\eta}(t)^{*} \cdot \boldsymbol{\eta}(t)\right)
\end{aligned}
$$

and then finally Eq.(56) yields the gauge fixed action

$$
S_{G F}=S[\mathbf{V}, \boldsymbol{\sigma}]+\int d t\left\{\frac{1}{2 \xi} \mathbf{V}_{\mathbf{0}}^{2}(t)+i \boldsymbol{\eta}^{*}(t) \cdot \boldsymbol{\eta}(t)\right\},
$$

to be contrasted with Eq.(57). The net result of this alternative choice of gauge function is to remove the time derivative from the Grassmann fields in the gauge fixed action Eq.(75).

In this latter case, the BRS transformation that leaves the gauge-fixed action Eq.(75) invariant includes in part the infinitesimal extended Galilean transformation obtained by replacing the variable boost velocity by the time dependent Grassmann vector $\mathbf{c}(t) \rightarrow \zeta \boldsymbol{\eta}(t)$, where $\zeta$ is the real Grassmann constant we introduced before. Namely,

$$
\begin{aligned}
\delta_{\mathrm{BRS}} \mathbf{x} & =-\zeta \int_{0}^{t} d u \boldsymbol{\eta}(u) \\
\delta_{\mathrm{BRS}} t & =0 \\
\delta_{\mathrm{BRS}} \mathbf{V}(\mathbf{x}, t) & =\zeta \int_{0}^{t} d u \boldsymbol{\eta}_{k}(u) \frac{\partial \mathbf{V}(\mathbf{x}, t)}{\partial x_{k}}-\zeta \boldsymbol{\eta}(t), \\
\delta_{\mathrm{BRS}} \boldsymbol{\sigma}(\mathbf{x}, t) & =\zeta \int_{0}^{t} d u \boldsymbol{\eta}_{k}(u) \frac{\partial \boldsymbol{\sigma}(\mathbf{x}, t)}{\partial x_{k}}, \\
\delta_{\mathrm{BRS}} \Pi(\mathbf{x}, t) & =\zeta \int_{0}^{t} d u \boldsymbol{\eta}_{k}(u) \frac{\partial \Pi(\mathbf{x}, t)}{\partial x_{k}}+\mathbf{x} \cdot \zeta \dot{\boldsymbol{\eta}}(t) .
\end{aligned}
$$

This version of the BRS transformation reduces to that found in [13] in the limit that EGI goes over to standard GI (zero frame acceleration). To complete the BRS transformation, we must specify how the velocity zero mode and Grassmann variables transform. These are simply

$$
\begin{aligned}
\delta_{\mathrm{BRS}} \mathbf{V}_{\mathbf{0}}(t) & =-\zeta \boldsymbol{\eta}(t), \\
\delta_{\mathrm{BRS}} \boldsymbol{\eta}(t) & =0 \\
\delta_{\mathrm{BRS}} \boldsymbol{\eta}^{*}(t) & =\frac{-i}{\xi} \mathbf{V}_{\mathbf{0}}(t) \zeta .
\end{aligned}
$$


Note: we could also choose the more "symmetric" assignment $\mathbf{c}(t) \rightarrow \zeta(\boldsymbol{\eta}(t)+\dot{\boldsymbol{\eta}}(t))$, and then make the associated simple adjustments to the above set of transformation rules, but this has no effect upon the generalized Ward identity that follows from BRS. To summarize so far, the gauge fixed action Eq.(775) is invariant under this BRS transformation Eqs(76, 83$)$.

To finish, we next subject $Z_{G F}$ in Eq.(67) to the above BRS transformation, where now $S_{G F}$ is given by $\mathrm{Eq(175)}$. Following the now rather familiar steps, this leads to the equation $\delta_{B R S} Z_{G F}=0$, and expressing this in terms of the effective action $\Gamma$, yields the Slavnov-Taylor identity

$$
\left[\frac{i}{\xi} \int d t \frac{\delta \Gamma}{\delta \boldsymbol{\eta}_{c l}^{*}(t)} \cdot \mathbf{V}_{\mathbf{0}}^{c l}(t)+\int d \mathbf{x} \int d t \rho_{j}^{c l}(t)\left(\frac{\delta \Gamma}{\delta V_{l}^{c l}} \nabla_{j} V_{l}^{c l}+\frac{\delta \Gamma}{\delta \sigma_{l}^{c l}} \nabla_{j} \sigma_{l}^{c l}+\frac{\partial}{\partial t} \frac{\delta \Gamma}{\delta V_{j}^{c l}}\right)\right]=0
$$

where we have defined $\boldsymbol{\rho}^{c l}(t)=\int_{0}^{t} d u \boldsymbol{\eta}^{c l}(u)$, so that $\dot{\boldsymbol{\rho}}^{c l}(t)=\boldsymbol{\eta}^{c l}(t)$. The Slavnov-Taylor identities that follow from EGI Eqs.(68,84) and the Ward identities that they imply Eqs.(69, 71) have not, to our knowledge, been derived nor considered before. From this point on, the entire discussion immediately following Eq.(68) remains intact and applies without modification to Eq.(84), as does the discussion concerning the implications of this identity Eq.(84) for vertex renormalization.

\section{WARD IDENTITIES IN QED AND IN THE STOCHASTIC FIELD THEORY OF RANDOMLY STIRRED FLUIDS}

The well-known Ward identity in QED relating the exact photon-electron vertex and the electron propagator reads,

$$
S_{F}^{\prime-1}(p+q)-S_{F}^{\prime-1}(p)=q^{\mu} \Gamma_{\mu}(p+q, p),
$$

where $p+q, q$ denote the four-momentum of the entering and emerging electron lines, respectively, $\Gamma_{\mu}$ denotes the exact photon-fermion-fermion vertex and $S_{F}^{\prime}(k)$ is the exact Feynman fermion propagator [27]. This identity allows us to compute $S_{F}^{\prime}$ directly from knowledge of the vertex and is represented graphically in Eq.(86) where the blobs denote the exact expressions. The four-momenta carried by the individual fermion lines (single arrowed-lines) is indicated by the quantities within parentheses. Note that the four-momenta carried by the photon line (wiggly) on the right-hand side of Eq.(86) is therefore $=q$. This identity is obviously satisfied by the bare vertex $\Gamma_{\mu}=\gamma_{\mu}$ and bare propagator, $S_{F}(p)=\left(p_{\mu} \gamma^{\mu}-m\right)^{-1}$. 
$(p+q) \longrightarrow-(p) \longrightarrow=(p+q) \longrightarrow$

Taking the $q \rightarrow 0$ limit of Eq.(85) yields

$$
\frac{\partial S_{F}^{\prime-1}(p)}{\partial p^{\mu}}=\Gamma_{\mu}(p, p),
$$

which holds for the special case when the momentum transfer to the photon approaches zero. The crux of this identity is the observation that the derivative of a fermion line with respect to the four-momentum is equivalent to the insertion of a photon line in the limit of zero momentum transfer. This derivative rule therefore generates the "soft photon" vertex in QED. We illustrate this differential identity Eq.(87) diagramatically in Eq.(88): note that the four-momenta of the photon line (wiggly) is zero: $q=0$.

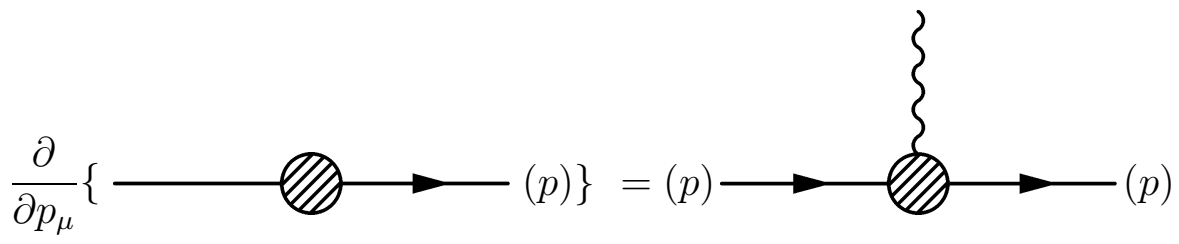

Having briefly reviewed the main features of the key Ward identity in QED [27], we come back to the analogous identities that hold in the stochastic field theory of the randomly forced NSE. The exact identity Eq.(71) is shown in diagram form in Eq.(89) and holds for the exact inverse response function and triple vertex (indicated with blobs). The zig-zag and arrowed double lines denote the conjugate and velocity fields, respectively. The momentum and frequency carried by the various lines is indicated by the associated pair of variables $(\boldsymbol{k}, \omega)$. Note that the momentum and frequency carried by the vertical velocity line (arrowed double line) on the right-hand side of Eq. (89) is therefore $=(\mathbf{0}, \omega)$. This identity is obviously satisfied by the bare inverse response function $\Gamma_{i j}^{(1,1)}(\boldsymbol{k}, \omega)=\left(-i \omega+\nu_{0} k^{2}\right) P_{i j}(\boldsymbol{k})$ and bare vertex $\Gamma_{n i j}^{(2,1)}=i k_{n} P_{i j}(\boldsymbol{k})[4]$. This identity is structurally quite similar to the QED Ward 
identity in (85) but there is an important difference: in Eq.(71) the 3-momentum transfer to the inserted velocity line is always zero, whereas any finite frequency $\omega$ can be transferred.

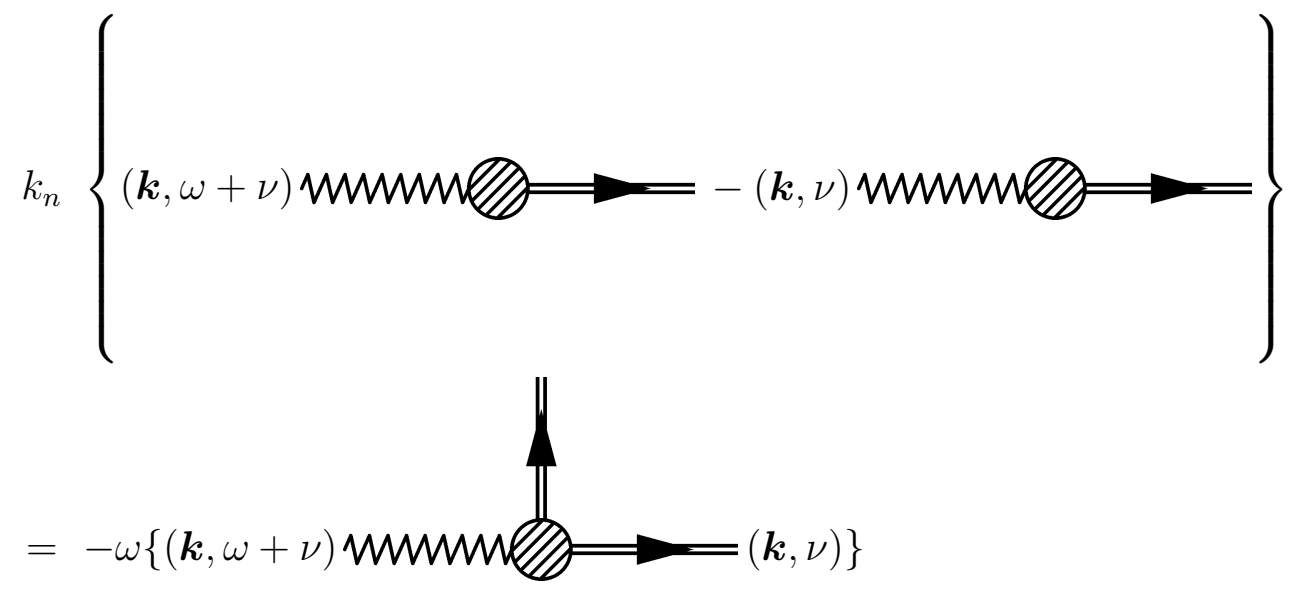

Taking the zero-frequency limit $(\omega \rightarrow 0)$ yields the further differential identity in Eq.(72) which as we see, holds for the special case when the frequency transfer to the inserted zeromomentum velocity line goes to zero. The crux of this identity is the simple observation that the derivative of the exact response function with respect to frequency is equivalent to the insertion of a velocity line in the limit of both vanishing momentum and frequency transfer: $=(\mathbf{0}, 0)$. We illustrate this differential identity in Eq.(90). This derivative operation therefore generates the "soft velocity" vertex in NSE diagrammatic perturbation theory: the momentum-frequency pair carried by the velocity (the vertical arrowed double line on the right-hand side of Eq.(90) ) is $=(\mathbf{0}, 0)$.

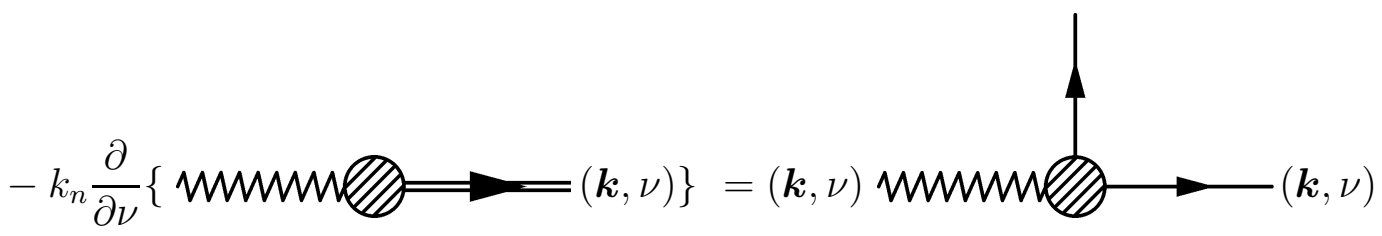

The rigid frame velocity, relative to the lab frame, is what enters into the WTI's for the NSE. This velocity can either be a constant, in which case we have the strict Galilean transformation, or it can be time dependent but rectilinear, in which case we have the extended Galilean transformation. This velocity enters the triple vertex as a background field. This is further supported by looking directly at the NSE in momentum space and time [28] and 
examining the nonlinear contribution. This contribution sums over all velocity modes and time dependences; there is a single contribution coming from the time dependent zero-mode which is simply the contribution coming from rigidly translating the reference frame. So the WTI is the statement that it is only this rigid motion that does not renormalize. This bulk system motion can correspond to a constant velocity, or a time dependent but rectilinear velocity, i.e., an arbitrary rectilinear acceleration.

Regarding our comparison to gauge field theory, this has a two-fold motivation. On the one hand, we note that the fluid velocity transformation $\mathbf{V} \rightarrow \mathbf{V}^{\prime}=\mathbf{V}+\mathbf{c}$ and the transformation of the $\mathrm{QED}$ vector potential $\mathbf{A} \rightarrow \mathbf{A}^{\prime}=\mathbf{A}+\nabla \phi$ are affine transformations leaving the convective derivative in the Navier-Stokes equation invariant and the $U(1)$ covariant derivative invariant, respectively. The second motivation is inspired in large part by the structure of the Ward identities that follow from breaking Galilean (or extended Galilean) invariance, since these identities Eqs.(71, 72) do closely resemble those that follow from breaking gauge invariance in QED, Eqs.(85,87), respectively, as we argued above. QED of course has a local (gauge) invariance, whereas Galilean invariance is a global symmetry.

One can also make an analogy between Galilean invariance and field theories with global symmetries. For example consider symmetry breaking in a globally invariant field theory such as N-component scalar field theory with a global $O(N)$ invariance, where many results have been obtained. Considering this case in the symmetry broken phase and shifting the field about the vacuum expectation value $\mathbf{v} \equiv\langle\phi\rangle$, one can derive Ward identities involving v. In particular, there is an identity relating the two and three point 1PI functions (vertices) that can be compared to the one we derived for broken GI or for broken EGI. This is (for complete details refer to [8]),

$$
t_{l i}^{\alpha} \Gamma_{i k}^{(2)}(p)+t_{k i}^{\alpha} \Gamma_{i l}^{(2)}(p)+v_{j} t_{j i}^{\alpha} \Gamma_{i k l}^{(3)}(p)(0, p,-p)
$$

where the $t^{\alpha}, \alpha=1,2, \ldots, N$ are $N \times N$ real antisymmetric matrices: the generators of the $O(N)$ Lie algebra. These of course have no analogue in our field theory, as the Galilean transformation is abelian, whereas $O(N)$ is a nonabelian symmetry group. Apart from these important Lie algebra factors, this identity holds for the triple vertex $\Gamma^{(3)}$ at zero fourmomentum transfer, an important distinction to Eq.(171) which holds for zero 3-momentum transfer but for arbitrary finite frequency transfer. From the asymmetry of the $t^{\alpha}$ we can write $t_{k i}^{\alpha}=-t_{i k}^{\alpha}$ and so express the first two terms in Eq.(91) as a difference, but there is no 
obvious limit to take here that would yield even a formal derivative, thus making comparison to the zero-momentum and zero frequency transfer Ward identity in Eq.(72) difficult. From these considerations, we conclude that the Ward identities resulting from broken Galilean and broken extended Galilean invariance bear a closer resemblance to those arising in QED, than to the those from the above globally invariant N-component scalar field theory.

\section{IMPLICATIONS FOR VERTEX RENORMALIZATION}

The vertex function $\Gamma^{(2,1)}$ is associated with the nonlinear or advective term in the NavierStokes equation. In the action formalism Eq.(2), this vertex is given at tree-level by the nonlinear or advective term multiplied by the conjugate field: $\sigma_{k} P_{k j}(\nabla) \frac{\partial\left(V_{l} V_{j}\right)}{\partial x_{l}}$. It is thus a three-legged object, built up from one conjugate field and two velocity fields, and this fact is reflected when writing out the three arguments of wavevector-frequency pairs. Of course, both wavevector and frequency are independently conserved at the vertex, so it is in general a function of two independent wavevector-frequency pairs (see e.g., Ref.[4] for the elements of Navier-Stokes diagrammatic perturbation theory). The most general form of the vertex taking into account corrections and possible renormalization effects must reflect this fact. We can separate out the tree level or zero loop contribution, and thus the term $\Lambda$ represents all the possible (higher-loop) corrections:

$$
\Gamma_{i j k}^{(2,1)}(\mathbf{q}, \omega ; \mathbf{k}, \nu ; \mathbf{q}-\mathbf{k}, \omega-\nu)=i k_{i} P_{j k}(\mathbf{k})+\Lambda_{i j k}(\mathbf{q}, \omega ; \mathbf{k}, \nu ; \mathbf{q}-\mathbf{k}, \omega-\nu) .
$$

In Eq.(92) our convention is that the middle argument corresponds to the incoming wavevector and frequency carried by the conjugate field, whereas the first and third arguments correspond to the wavevector/frequency pair carried by the two velocity fields that meet at the vertex.

In a similar fashion, the exact inverse response function can be written as follows:

$$
\Gamma_{j k}^{(1,1)}(\mathbf{k}, \omega ;-\mathbf{k},-\omega)=\left[-i \omega+\bar{\nu}(\omega, k) k^{2}+\Sigma(\omega, k)\right] P_{j k}(\mathbf{k}),
$$

where $\bar{\nu}(\omega, k)$ and $\Sigma(\omega, k)$ denote a renormalized viscosity and a "mass" term, respectively. At tree level, this corresponds to the term in the action $\sigma_{k}\left(\frac{\partial V_{k}}{\partial t}-\nu_{0} \nabla^{2} V_{k}\right)$. So, the inverse response function $\Gamma^{(1,1)}$, is a two-point object (built from one conjugate and one velocity field) and thus a function of just one wavevector and one frequency [4]. The Ward identities 
derived above in Eqs.(71,72) impose certain constraints on these three functions $\bar{\nu}, \Sigma$ and $\Lambda$. In other words, they relate the nonlinear and linear parts of the Navier-Stokes equation. First consider implications of extended Galilean invariance. Inserting Eqs.(92,93) into Eq.(171) implies that

$$
\begin{aligned}
& k_{n} P_{j k}(\mathbf{k}) \quad\left[(\bar{\nu}(\omega+\nu, k)-\bar{\nu}(\nu, k)) k^{2}+(\Sigma(\omega+\nu, k)-\Sigma(\nu, k))\right] \\
& =-\omega \Lambda_{n j k}(\mathbf{0}, \omega ; \mathbf{k}, \nu ;-\mathbf{k}, \omega-\nu) .
\end{aligned}
$$

Thus, a vertex correction (i.e., right-hand side of Eq(94)) can arise provided that the renormalized viscosity and/or the mass term are frequency dependent functions, according to the left-hand side of this relation. However, recall that the reference system is being subject to arbitrary rectilinear accelerations, and this noninertial bulk motion does introduce an explicit time (and hence frequency) dependence into the system. Nevertheless, the correction to the vertex in this case is the specific one indicated in Eq.(94). We see that the vertex correction that enters into this relation is the one for which one of the two velocity legs carries zero wavevector $\mathbf{0}$ but finite frequency $\omega$, i.e., this leg corresponds to a time-dependent velocity zero-mode.

Next, we come to the implication of Galilean invariance. This can be had by either inserting Eqs.92 93) into Eq.(72) or by taking the zero frequency limit $\omega \rightarrow 0$ directly in Eq.(94). Either way, we obtain [7]

$$
k_{n} \frac{\partial}{\partial \nu}\left[\bar{\nu}(\nu, k) k^{2}+\Sigma(\nu, k)\right] P_{j k}(\mathbf{k})=-\Lambda_{n j k}(\mathbf{0}, 0 ; \mathbf{k}, \nu ;-\mathbf{k},-\nu) .
$$

As stated in [7], as a model of stationary forced turbulence, neither the viscosity nor the mass term will depend on frequency, so that for stationary random forcing, the constraint Eq.(95) implies that

$$
\Lambda_{n j k}(\mathbf{0}, 0 ; \mathbf{k}, \nu ;-\mathbf{k},-\nu)=0 .
$$

This specific vertex correction is in fact zero, and it corresponds to the situation in which one of the two velocity legs carries zero wavevector $\mathbf{k}=\mathbf{0}$ and zero frequency $\omega=0$, and this corresponds to a velocity zero mode.

These considerations are important. There has been a longstanding question about the range of validity of the vertex Ward identity and the vertex non-renormalization property it implies [2, 3, 4, 6, 7, 11, 12, 13]. In [13], we made a definitive resolution of this problem by discovering that the dynamic functional for Navier-Stokes theory possesses an underlying 
and fundamental BRS symmetry. Since the fields of the BRS symmetry involve only the zero mode, that demonstration makes explicit that the associated vertex Ward identity is valid only at exactly zero momentum transfer $\mathbf{k}=\mathbf{0}$, and not in some small limiting region (i.e., for $k \rightarrow 0$ ) around this. The statement that vertex renormalization is not constrained by Galilean invariance was put forward somewhat earlier in [12], using physical arguments and employing the Reynolds decomposition. Our derivation in [13] validates the assertions made

in [12] through a mathematically precise and complete construction (see statements in Ref. [5] concerning the mathematical rigor of the path integral formalism). Except for the zero mode, neither Galilean nor extended Galilean invariance constrain vertex renormalization.

\section{DISCUSSION}

It has been known for a long time that the randomly forced Navier-Stokes (NSE) equation can be cast in terms of a path integral [1, 2, 3, 4, 5, 6, 7, 9]. The underlying symmetries of this equation can then be treated at the level of the functional integral. In particular, the Galilean invariance of the NSE is formally analogous to global gauge invariance in quantum field theory. The well established methods for gauge fixing can then be brought to bear and used to restrict the sum over histories of Navier-Stokes dynamics to pick out one inertial frame. This gauge fixing was in fact used recently to eliminate an infinite number of spurious correlation functions that are implied by the non-gauge fixed functional [13]. In addition, the evaluation of non-Galilean invariant quantities (e.g., n-point correlations of velocity fields) in the standard path integral Eq.(1) leads to over-counting of configurations and spurious relations, so we must fix the gauge, that is, break the Galilean invariance. If in the future, simulations are carried out using such functionals, our gauge-fixing procedure could possibly provide some numerical advantages in allowing for a faster and more efficient code. However, once the functional has been gauge-fixed, it is crucial to ascertain whether the well known and oft-cited Ward identity for the inverse response and vertex functions [2, 3, 4, 6, 7, 11, 12] remains valid or not. Regarding this question, it is important to recognize that the gaugefixed theory possesses a Becchi-Rouet-Stora (BRS) symmetry [20] which when brought out and exposed, restores a type of hidden Galilean invariance. The celebrated Ward identity is therefore re-established, but it is now understood to be a direct consequence of this BRS invariance, and not of the original Galilean invariance. 
We emphasize that our BRS symmetry follows from gauge fixing and should not be confused with other kinds of BRS invariance, such as those treated for example, in chapter 16 of Zinn-Justin's book [8]. In these latter cases, it is the stochastic differential equation itself that is regarded as a local constraint equation for the field variable. The determinant of the Jacobian of this stochastic equation is then expressed as a Grassmann integral over ghost fields in the path integral representation of the generating functional for the correlation functions. An effective action is then identified, and it is this action that is shown to possess a simple kind of BRS symmetry, and sometimes even a kind of supersymmetry. This differs distinctively from our treatment here, where by contrast, the BRS symmetry emerges from an attempt to correct an infinity in the NSE dynamic functional arising from the underlying Galilean (and extended Galilean) invariance of the theory. The ghost and BRS formalism provides the natural language in which to establish the Ward identities. We are not required to "compensate" any degrees of freedom as in quantum gauge theories. Our use of the ST identities in the stochastic field theory of randomly stirred flows has an entirely different significance to the one encountered in quantum field theories.

The NSE is invariant as well under rectilinear but otherwise arbitrary frame accelerations. This extended Galilean invariance (EGI) has been considered previously in various differing contexts [22, 23, 24, 25]. Just as for Galilean invariance, EGI can be regarded as a gauge symmetry at the level of the path integral. This higher gauge symmetry can be fixed, which corresponds to choosing one rectilinearly accelerating reference frame. Just as before, the gauge fixed theory possesses an underlying BRS symmetry which when invoked, leads to a generalized Ward identity for the inverse response and vertex functions. This new Ward identity reduces to the standard one in the limit of zero frame acceleration. It contains additional information regarding the vertex renormalization implied by arbitrary rectilinear frame accelerations, that is not provided by the "standard" Ward identity. Extended Galilean invariance is of interest in its own right, for by the principle of equivalence [29], it corresponds to the invariance of the stirred flows under arbitrary time-dependent unidirectional background gravitational fields.

The standard Ward identity has been appealed to on numerous occasions to make statements about the nonrenormalization of the advective or inertial term in the Navier-Stokes equation [2, 3, 4 4, 6, 7, 11, 12]. Recently, McComb claimed that Galilean invariance does not at all constrain the vertex renormalization [12]. To quote his paper verbatim, "Galilean 
invariance has been used as the justification of Ward identities, which in turn lead to the conclusion that in the perturbative renormalization group (RG) the vertex is not renormalized". Nevertheless, it is argued in Ref.[12] that "vertex renormalization is not constrained by this (Galilean) symmetry". Section $\mathrm{V}$ of this paper spells out explicitly what both the "standard" and the generalized Ward identities imply for the corrections to the bare, tree-level vertex. We emphasize there how our results, derived from field theory methods, complement, validate and confirm the assertions made in Ref [12]. To test the claim put forward in [12], one must go back to the Ward identity, as well as its generalized version, and examine carefully what constraints are actually imposed by them. The field-theoretic formalism employed here is needed to work out the consequences of breaking both Galilean invariance and its extension. The final goal of this is to understand the vertex renormalization problem. The constraints on the vertex that follow from Galilean invariance and from extended Galilean invariance (more precisely, that follow from the respective BRS symmetries of the gauge-fixed theories) are quite weak. In the case of Galilean invariance, the vertex correction that "couples" to the zero mode of the system is constrained to be zero. For EGI, the vertex correction that involves the system's bulk acceleration is computable in terms of the frequency dependent viscosity and mass terms of the renormalized inverse response function. Galilean invariance and EGI constrain only the spatial zero mode of the vertex but none of the higher wavenumber modes. This information is new and impacts directly on the physics of randomly stirred flows. These points can be appreciated from the diagrammatic comparison of the corresponding identities from QED and the field theory of the randomly stirred NSE in Sec IV.

It is possible to use the functional Eq(1) without explicitly fixing the zero-mode for deriving perturbative expansions, as has been done up to now. Thus by implicitly fixing the zero mode, the perturbation expansions from the functional are consistent with those based directly on the NS equation itself. However, in looking for exact relations between correlation functions from the functional, unless one is careful, the problem of the zero mode then shows up and can lead to incorrect results. This mistake precisely underlies the confusion that has been generated over three decades in regards vertex renormalization claims. We have demonstrated here, following on from [13], how to explicitly fix the zero mode via the analogy we identified with gauge fixing, and thus obtain a formally well defined functional. 
Although we focussed attention here exclusively on the randomly forced Navier-Stokes equation, the gauge fixing procedure in this paper can be applied to other Galilean invariant theories such as the KPZ equation of random surface growth [30], magnetohydrodynamics and the stochastic Burgers equation [31]. In [31], a saddle point approximation is applied to the path integral for the Burgers equation to calculate the tails of the probability density function for the velocity. A special feature of that problem is a symmetry of gauge-invariance type. In fact, the Burgers action is seen to be invariant under an extended Galilean transformation. This gauge degree of freedom is then integrated over by the standard FaddeevPopov trick. The aim in [31] was to show that the fluctuations around the instanton are free from infrared divergences. Once the action for the Burgers equation is gauge fixed and its EGI broken in this way, then there should be no spurious correlators there either. We moreover conjecture that there is a corresponding BRS symmetry that restores the EGI of the gauge-fixed Burgers dynamic functional with a subsequent Ward identity following as a consequence of this symmetry.

A few comments regarding our use of gauge-fixing terminology are in order. With respect to the terminology as employed in (quantum) field theory, a local symmetry is one in which the associated field transformation involves parameters that depend on the spatial coordinate x. This is not the case for the Galilean transformation Eqs. (36 6), and so it is correct to classify this as a global (or rigid) transformation. The Galilean transformation is a global space and time transformation between two separate inertial frames of reference. In standard field theory texts (see e.g., [8] and [10]) the local transformations are also denoted as gauge transformations. Even though Galilean invariance is a global symmetry, we find it to useful to employ the terminology of gauge invariance and gauge fixing in this paper, especially since our breaking of this global symmetry can be handled with exactly the same methods (FP determinant, BRS, etc), as employed in gauge theories. Moreover, for extended Galilean invariance, the associated transformation is indeed local in time and so does resemble more closely a gauge theory. Note also that the convective derivative in the NS equation is invariant under a Galilean transformation, see e.g., the left hand side of Eq.(48). This reminds one of the invariance of the $\mathrm{U}(1)$ covariant derivative under a gauge transformation, where for EGI, the function $\lambda(t)$ plays the role of the arbitrary gauge function of QED. Since the steps for symmetry breaking in the path integral very closely resemble the methods employed for gauge fixing (see Appendix A), and since a BRS 
invariance results with all its implications for Ward identities, we feel that it is useful and instructive to use the terminology of broken gauge invariance in this paper, albeit perhaps in a slightly loose manner.

In summary this work and [13] have uncovered a new symmetry in the stirred NSE and for related Galilean invariant stochastic equations. Exploiting this symmetry may be useful in performing Monte Carlo simulations of the path integrals and actions corresponding to these systems [14, 32]. Moreover, given the deep significance of symmetries in physical problems, other applications may benefit from recognizing this invariance.

\section{Acknowledgments}

Support was provided to A.B. by the UK Science and Technology Facilities Council (STFC) and D.H. acknowledges the Grant AYA2006-15648-C02-02 from the Ministerio de Ciencia e Innovación (Spain).

\section{APPENDIX A: GAUGE FIXING}

The steps outlined here closely parallel those used for gauge fixing in quantum field theories [26] and are adapted to the case at hand. We first introduce the functional $\Delta_{f}[\mathbf{V}]$ by the following equation:

$$
1=\Delta_{f}[\mathbf{V}] \int d \mathbf{c} \delta\left[\mathbf{f}\left(\mathbf{V}^{\mathbf{c}}\right)\right]
$$

and $\mathbf{V}^{\mathbf{c}}$ denotes the result of the Galilean transformation (GT) applied to the velocity field V; see Eq.(3), and the integral is over all constant boost velocities. We assume that the equation $\mathbf{f}\left(\mathbf{V}^{\mathbf{c}}\right)=0$ has exactly one solution $\mathbf{c}$ for any initial configuration $\mathbf{V}$. If we apply a second GT, i.e. we next transform to a new inertial frame (double-prime) moving with a velocity $\mathbf{b}$ with respect to the primed frame, then the transformation rules Eqs.(3-6] tell us that $\mathbf{x}^{\prime}=\mathbf{x}^{\prime \prime}+\mathbf{b} t^{\prime}$ and $t^{\prime}=t^{\prime \prime}$ and

$$
\begin{aligned}
\left(\mathbf{V}^{\mathbf{c}}\right)^{\mathbf{b}}(\mathbf{x}, t) & =\left(\mathbf{V}^{\prime}\left(\mathbf{x}^{\prime}, t^{\prime}\right)+\mathbf{c}\right)^{\mathbf{b}} \\
& =\mathbf{V}^{\prime \prime}\left(\mathbf{x}^{\prime \prime}, t^{\prime \prime}\right)+\mathbf{c}+\mathbf{b} \\
& =\mathbf{V}^{\prime \prime}(\mathbf{x}-(\mathbf{c}+\mathbf{b}) t, t)+\mathbf{c}+\mathbf{b}=\left(\mathbf{V}^{\mathbf{b}}\right)^{\mathbf{c}}(\mathbf{x}, t) \equiv \mathbf{V}^{\mathbf{c}+\mathbf{b}}
\end{aligned}
$$


This exercise is needed to prove that the Fadeev-Popov (FP) determinant $\Delta_{f}[\mathbf{V}]$ is invariant. The invariance of the FP determinant is needed in turn, to ensure that the volume of the "gauge-group" orbit can be factored out from the functional (see, e.g., [33]). The first requirement follows from Eq.(A1) using Eq.(A2):

$$
\Delta_{f}^{-1}\left[\mathbf{V}^{\mathbf{b}}\right]=\int d^{3} \mathbf{c} \delta^{3}\left(\mathbf{f}\left(\left(\mathbf{V}^{\mathbf{b}}\right)^{\mathbf{c}}\right)\right)=\int d^{3}(\mathbf{c}+\mathbf{b}) \delta^{3}\left(\mathbf{f}\left(\mathbf{V}^{\mathbf{c}+\mathbf{b}}\right)\right)=\Delta_{f}^{-1}[\mathbf{V}]
$$

Then, repeating the standard manipulations [26], we can prove that the volume of the gauge group indeed factorizes out to produce an overall infinite constant. Insert Eq.(A1) into the functional Eq.(11) apply a Galilean transformation, then using Eq.(A3) we obtain

$$
\left(\int d \mathbf{c}\right) \int[D \mathbf{V}][D \boldsymbol{\sigma}] \Delta_{f}[\mathbf{V}] \delta[\mathbf{f}(\mathbf{V})] \exp \{-S[\mathbf{V}, \boldsymbol{\sigma}]\}
$$

We still need to actually calculate the FP determinant. To calculate it, recall [33] it is sufficient to do so for infinitesimal "gauge"-transformations (so, infinitesimal GT's):

$$
\Delta_{f}[\mathbf{V}]=\left.\operatorname{det} \frac{\delta f\left(\mathbf{V}^{\mathbf{c}}\right)}{\delta \mathbf{c}}\right|_{\mathbf{c}=\mathbf{0}}=\left.\operatorname{det}\left(\frac{\partial f_{i}}{\partial c_{j}}\right)\right|_{\mathbf{c}=\mathbf{0}}
$$

We remark that the FP determinant Eq.(A5) is an ordinary discrete matrix determinant: it is not a functional determinant. This is because the gauge parameter (= boost velocity) is an ordinary constant vector, not a field. Next, we consider the class of "gauge" conditions of the form $\mathbf{f}(\mathbf{V})-\mathbf{U}(\mathbf{x}, t)=0$, where $\mathbf{U}(\mathbf{x}, t)$ is an arbitrary function. The FP determinant is as before because $\mathbf{U}(\mathbf{x}, t)$ is unaffected by a GT. We make use of this feature to replace the delta function in Eq.(A4) by some other function(al) which may be more convenient for practical calculations. So, in this gauge the path integral Eq. (A4) becomes

$$
\left(\int d \mathbf{c}\right) \int[D \mathbf{V}][D \boldsymbol{\sigma}] \Delta_{f}[\mathbf{V}] \delta[\mathbf{f}(\mathbf{V})-\mathbf{U}(\mathbf{x}, t)] \exp \{-S[\mathbf{V}, \boldsymbol{\sigma}]\}
$$

This expression is independent of $\mathbf{U}(\mathbf{x}, t)$, so we can integrate over an arbitrary weight functional. As usual, a popular choice is the exponential [26]:

$$
G[\mathbf{U}]=\exp \left(-\frac{1}{2 \xi} \int d \mathbf{x} d t \mathbf{U}^{2}(\mathbf{x}, t)\right)
$$

for real parameter $\xi>0$. For the final step, integrate Eq.(A6) over $\mathcal{D} \mathbf{U}$ to obtain

$$
\int[D \mathbf{V}][D \boldsymbol{\sigma}] \Delta_{f}[\mathbf{V}] \exp \left\{-S[\mathbf{V}, \boldsymbol{\sigma}]-\frac{1}{2 \xi} \int d \mathbf{x} d t(\mathbf{f}(\mathbf{V}))^{2}\right\}
$$

This is the gauge fixed dynamic functional for randomly stirred incompressible fluids expressed for the gauge function $\mathbf{f}$. 


\section{APPENDIX B: EXPANSION FOR $\Gamma$}

We write out the first few terms of the functional Taylor series for the effective action in Eq. (27). We display only those terms actually needed to derive the Ward identity in Eq. (28) in this paper. In wavevector and frequency space these are:

$$
\begin{aligned}
& \Gamma\left[\mathbf{V}^{c l}, \sigma^{c l}\right]=\cdots \int d \mathbf{q}_{1} d \omega_{1} \int d \mathbf{q}_{2} d \omega_{2} V_{\alpha}^{c l}\left(\mathbf{q}_{1}, \omega_{1}\right) \sigma_{\beta}^{c l}\left(\mathbf{q}_{2}, \omega_{2}\right) \Gamma_{\alpha \beta}^{(1,1)}\left(-\mathbf{q}_{1},-\omega_{1} ;-\mathbf{q}_{2},-\omega_{2}\right) \\
+ & \frac{1}{2} \int d \mathbf{q}_{1} d \omega_{1} \int d \mathbf{q}_{2} d \omega_{2} \int d \mathbf{q}_{3} d \omega_{3} V_{\alpha}^{c l}\left(\mathbf{q}_{1}, \omega_{1}\right) V_{\beta}^{c l}\left(\mathbf{q}_{2}, \omega_{2}\right) \sigma_{\gamma}^{c l}\left(\mathbf{q}_{3}, \omega_{3}\right) \times \\
& \Gamma_{\alpha \beta \gamma}^{(2,1)}\left(-\mathbf{q}_{1},-\omega_{1} ;-\mathbf{q}_{2},-\omega_{2} ;-\mathbf{q}_{3},-\omega_{3}\right) \\
+ & \ldots
\end{aligned}
$$

\section{APPENDIX C: SPACE-TIME BOX}

The action and dynamic functional can be regularized by enclosing the system in a spatially and temporally bounded space-time box. This regularization admits a corresponding discrete version of Galilean invariance, implying the box Ward identity derived above in Sec IIE from which we can demonstrate the appearance of spurious relations, in complete parallel to the continuum case treated in [13]. Our box is defined from coordinates $x=0-L$, $y=0-L$, and $z=0-L$ and time defined from $t=0-T$, subject to periodic boundary conditions in space and time.

We expand the fields as

$$
\begin{aligned}
v_{\alpha}(\mathbf{x}, t) & =\sum_{\mathbf{n}, j}\left(\frac{1}{L}\right)^{3} \frac{1}{T} \tilde{v}_{\alpha}(\mathbf{k}, \omega) \exp \left[i \frac{2 \pi}{L}\left(n_{1} x_{1}+n_{2} x_{2}+n_{3} x_{3}\right)-i \frac{2 \pi}{T} j t\right], \\
\sigma_{\alpha}(\mathbf{x}, t) & =\sum_{\mathbf{n} \cdot j}\left(\frac{1}{L}\right)^{3} \frac{1}{T} \tilde{\sigma}_{\alpha}(\mathbf{k}, \omega) \exp \left[i \frac{2 \pi}{L}\left(n_{1} x_{1}+n_{2} x_{2}+n_{3} x_{3}\right)-i \frac{2 \pi}{T} j t\right],
\end{aligned}
$$

and

$$
D_{\alpha \beta}(\mathbf{x})=\sum_{\mathbf{n}}\left(\frac{1}{L}\right)^{3} \tilde{D}_{\alpha \beta}(\mathbf{k}) \exp \left[i \frac{2 \pi}{L}\left(n_{1} x_{1}+n_{2} x_{2}+n_{3} x_{3}\right)\right] .
$$

The following integrals over the box are used below:

$$
\begin{aligned}
\int_{0}^{T} d t \exp \left[-i \frac{2 \pi}{T} j t\right] & =T \delta_{j, 0} \\
\int_{0}^{L} d^{3} \mathbf{x} \exp \left[i \frac{2 \pi}{L} \mathbf{n} \cdot \mathbf{x}\right] & =L^{3} \delta_{\mathbf{n}, \mathbf{0}} .
\end{aligned}
$$


In $\mathbf{k}, \omega$ space we will express the velocity and auxiliary fields in terms of dimensionless quantities as

$$
\begin{gathered}
\tilde{v}_{\alpha}(\mathbf{k}, \omega)=v_{\alpha}(\mathbf{n}, j) L^{4} \\
\tilde{\sigma}_{\alpha}(\mathbf{k}, \omega)=\sigma_{\alpha}(\mathbf{n}, j) \frac{T^{2}}{L},
\end{gathered}
$$

and

$$
\tilde{D}_{\alpha \beta}(\mathbf{k})=D_{\alpha \beta}(\mathbf{n}) \frac{L^{5}}{T^{3}}
$$

For purposes of dimensional counting, let the spatial dimensions be expressed as $d(x)=L$ and temporal as $d(t)=T$, which also means that $d(k)=L^{-1}$ and $d(\omega)=T^{-1}$. The action is dimensionless $d(S)=0$. Then from examining individual terms in the action we find $d(\tilde{v}(\mathbf{k}, \omega))=L^{4}, d(\tilde{\sigma}(\mathbf{k}, \omega))=T^{2} / L$ and $d(\nu)=L^{2} / T$. In configuration space $d(v(\mathbf{x}, t))=L / T$ and $d(\sigma(\mathbf{x}, t))=T / L^{4}$.

Thus the action Eq.(2) in discrete wavenumber and frequency coordinates is:

$$
\begin{aligned}
S & =\frac{1}{2} \sum_{\mathbf{n}} \sum_{j} \sigma_{\alpha}(-\mathbf{n},-j) D_{\alpha \beta}(\mathbf{n}) \sigma_{\beta}(\mathbf{n}, j) \\
& -i \sum_{\mathbf{n}} \sum_{j} \sigma_{\alpha}(-\mathbf{n},-j)\left[\left(-2 \pi i j+\bar{\nu} n^{2}\right) v_{\alpha}(\mathbf{n}, j)\right. \\
& \left.-2 \pi \bar{M}_{\alpha \beta \gamma}(\mathbf{n}) \sum_{\mathbf{m}} \sum_{l} v_{\beta}(\mathbf{n}-\mathbf{m}, j-l) v_{\gamma}(\mathbf{m}, l)\right],
\end{aligned}
$$

where we defined the dimensionless viscosity $\bar{\nu}$ as $\bar{\nu}=\nu_{0}(2 \pi)^{2} T / L^{2}$ and

$$
\bar{M}_{\alpha \beta \gamma}(\mathbf{n})=\frac{1}{2 i}\left[n_{\beta} \bar{P}_{\alpha \gamma}(\mathbf{n})+n_{\gamma} \bar{P}_{\alpha \beta}(\mathbf{n})\right],
$$

where

$$
\bar{P}_{\alpha \beta}(\mathbf{n})=\delta_{\alpha \beta}-\frac{n_{\alpha} n_{\beta}}{n^{2}} .
$$

In the equation Eq. (C9), everything is dimensionless.

The Fourier transform of the Galilean transformation between prime and unprimed frames, analogous to Eqs.(3-6), and expressed in discrete dimensionless coordinates is

$$
\begin{aligned}
\mathbf{n}^{\prime} & =\mathbf{n}, \\
j^{\prime} & =j-\overline{\mathbf{c}} \cdot \mathbf{n}, \\
v_{\alpha}(\mathbf{n}, j) & =v_{\alpha}^{\prime}(\mathbf{n}, j-\overline{\mathbf{c}} \cdot \mathbf{n})+\bar{c}_{\alpha} \delta^{3}(\mathbf{n}) \delta(j), \\
\sigma_{\alpha}(\mathbf{n}, j) & =\sigma_{\alpha}^{\prime}(\mathbf{n}, j-\overline{\mathbf{c}} \cdot \mathbf{n}),
\end{aligned}
$$


where we defined the dimensionless boost velocity $\overline{\mathbf{c}}$ as $\mathbf{c}=\overline{\mathbf{c}} L / T$. In general $\overline{\mathbf{c}} \cdot \mathbf{n}$ must be an integer for all integers $\mathbf{n}$, which thus means all three coordinates of the discretized boost velocity $\overline{\mathbf{c}}$ must be integers.

With the discrete Galilian transformations Eq. (ㄷ12) in hand we demonstrate that the discrete action Eq. (C9) is invariant. Begin with the term

$$
\begin{aligned}
& \sum_{\mathbf{m}} \sum_{l} v_{\beta}(\mathbf{n}-\mathbf{m}, j-l) v_{\gamma}(\mathbf{m}, l) \stackrel{\text { Gal-Trans }}{\Rightarrow} \\
= & \sum_{\mathbf{m}} \sum_{l} v_{\beta}^{\prime}(\mathbf{n}-\mathbf{m}, j-l-\overline{\mathbf{c}} \cdot[\mathbf{n}-\mathbf{m}]) v_{\gamma}^{\prime}(\mathbf{m}, l-\overline{\mathbf{c}} \cdot \mathbf{m}) \\
+ & \bar{c}_{\gamma} v_{\beta}^{\prime}(\mathbf{n}, j-\overline{\mathbf{c}} \cdot \mathbf{n})+\bar{c}_{\beta} v_{\gamma}^{\prime}(\mathbf{n}, j-\overline{\mathbf{c}} \cdot \mathbf{n})+\bar{c}_{\beta} \bar{c}_{\gamma} \delta(\mathbf{n}) \delta(j) .
\end{aligned}
$$

Therefore, we deduce the Galilean transformation of the following term in Eq.([C9):

$$
\begin{aligned}
& 2 \pi i \sum_{\mathbf{n}} \sum_{j} \sigma_{\alpha}(-\mathbf{n},-j) \bar{M}_{\alpha \beta \gamma}(\mathbf{n}) \sum_{\mathbf{m}} \sum_{l} v_{\beta}(\mathbf{n}-\mathbf{m}, j-l) v_{\gamma}(\mathbf{m}, l) \stackrel{\text { Gal-Trans }}{\Rightarrow} \\
= & 2 \pi i \sum_{\mathbf{n}} \sum_{j} \sigma_{\alpha}^{\prime}(-\mathbf{n},-j+\overline{\mathbf{c}} \cdot \mathbf{n}) \bar{M}_{\alpha \beta \gamma}^{\prime}(\mathbf{n})\left(\sum_{\mathbf{m}} \sum_{l} v_{\beta}^{\prime}(\mathbf{n}-\mathbf{m}, j-l-\overline{\mathbf{c}} \cdot[\mathbf{n}-\mathbf{m}]) v_{\gamma}^{\prime}(\mathbf{m}, l-\overline{\mathbf{c}} \cdot \mathbf{m})\right. \\
+ & \left.\bar{c}_{\gamma} v_{\beta}^{\prime}(\mathbf{n}, j-\overline{\mathbf{c}} \cdot \mathbf{n})+\bar{c}_{\beta} v_{\gamma}^{\prime}(\mathbf{n}, j-\overline{\mathbf{c}} \cdot \mathbf{n})+\bar{c}_{\beta} \bar{c}_{\gamma} \delta(\mathbf{n}) \delta(j)\right) \\
= & 2 \pi i \sum_{\mathbf{n}} \sum_{j^{\prime}} \sigma_{\alpha}^{\prime}\left(-\mathbf{n},-j^{\prime}\right) \bar{M}_{\alpha \beta \gamma}^{\prime}(\mathbf{n}) \sum_{\mathbf{m}} \sum_{l^{\prime}} v_{\beta}^{\prime}\left(\mathbf{n}-\mathbf{m}, j^{\prime}-l^{\prime}\right) v_{\gamma}^{\prime}\left(\mathbf{m}, l^{\prime}\right) \\
+ & 2 \pi \sum_{\mathbf{n}} \sum_{j^{\prime}} \sigma_{\alpha}^{\prime}\left(-\mathbf{n},-j^{\prime}\right)[\overline{\mathbf{c}} \cdot \mathbf{n}] v_{\alpha}^{\prime}\left(\mathbf{n}, j^{\prime}\right) .
\end{aligned}
$$

In arriving at the last equality, we have used fluid incompressibility $\mathbf{n} \cdot \mathbf{v}=0$, the fact that $\bar{M}_{\alpha \beta \gamma}^{\prime}(\mathbf{n}) \delta(\mathbf{n})=0$ as well as the change of discrete summation variables $j^{\prime}=j-\overline{\mathbf{c}} \cdot \mathbf{n}$ and $l^{\prime}=l-\overline{\mathbf{c}} \cdot \mathbf{m}$. Next, consider the Galilean transformation of the "propagator" term in Eq. (C9):

$$
\begin{aligned}
& -i \sum_{\mathbf{n}} \sum_{j} \sigma_{\alpha}(-\mathbf{n},-j)\left(-2 \pi i j+\bar{\nu} n^{2}\right) v_{\alpha}(\mathbf{n}, j) \stackrel{\text { Gal-Trans }}{\Rightarrow} \\
& =-i \sum_{\mathbf{n}} \sum_{j} \sigma_{\alpha}^{\prime}(-\mathbf{n},-j+\overline{\mathbf{c}} \cdot \mathbf{n})\left(-2 \pi i j+\bar{\nu} n^{2}\right)\left[v_{\alpha}^{\prime}(\mathbf{n}, j-\overline{\mathbf{c}} \cdot \mathbf{n})+\bar{c}_{\alpha} \delta(\mathbf{n}) \delta(j)\right] \\
& =-i \sum_{\mathbf{n}} \sum_{j} \sigma_{\alpha}^{\prime}(-\mathbf{n},-j+\overline{\mathbf{c}} \cdot \mathbf{n})\left(-2 \pi i j+\bar{\nu} n^{2}\right) v_{\alpha}^{\prime}(\mathbf{n}, j-\overline{\mathbf{c}} \cdot \mathbf{n}) \\
& =-i \sum_{\mathbf{n}} \sum_{j^{\prime}} \sigma_{\alpha}^{\prime}\left(-\mathbf{n},-j^{\prime}\right)\left(-2 \pi i j^{\prime}+\bar{\nu} n^{2}\right) v_{\alpha}^{\prime}\left(\mathbf{n}, j^{\prime}\right)-2 \pi \sum_{\mathbf{n}} \sum_{j^{\prime}} \sigma_{\alpha}^{\prime}\left(-\mathbf{n},-j^{\prime}\right)[\overline{\mathbf{c}} \cdot \mathbf{n}] v_{\alpha}^{\prime}\left(\mathbf{n}, j^{\prime}\right) .
\end{aligned}
$$


Lastly, the transformation of the "noise" term in Eq.([C9):

$$
\begin{aligned}
& \frac{1}{2} \sum_{\mathbf{n}} \sum_{j} \sigma_{\alpha}(-\mathbf{n},-j) D_{\alpha \beta}(\mathbf{n}) \sigma_{\beta}(\mathbf{n}, j) \stackrel{\text { Gal-Trans }}{\Rightarrow} \\
= & \frac{1}{2} \sum_{\mathbf{n}} \sum_{j} \sigma_{\alpha}^{\prime}(-\mathbf{n},-j+\overline{\mathbf{c}} \cdot \mathbf{n}) D_{\alpha \beta}^{\prime}(\mathbf{n}) \sigma_{\beta}^{\prime}(\mathbf{n}, j-\overline{\mathbf{c}} \cdot \mathbf{n}) \\
= & \frac{1}{2} \sum_{\mathbf{n}} \sum_{j^{\prime}} \sigma_{\alpha}^{\prime}\left(-\mathbf{n},-j^{\prime}\right) D_{\alpha \beta}^{\prime}(\mathbf{n}) \sigma_{\beta}^{\prime}\left(\mathbf{n}, j^{\prime}\right) .
\end{aligned}
$$

Adding up the transformed terms Eqs.C14,C15,C16) proves that the discrete action is indeed invariant under the discrete Galilean transformation $\mathrm{Eq}(\mathrm{C} 12)$.

[1] C. DeDominicis, J. Phys. (Paris) Colloq. 37 (1976) 247.

[2] C. DeDominicis and P.C. Martin, Phys. Rev. A19 (1979) 419.

[3] E.V. Teodorovich, J. Applied Math. and Mech. 53 (1989) 340.

[4] C-H. Mou and P.B. Weichman, Phys. Rev. E52 (1995) 3738.

[5] G.L. Eyink, J. Stat. Phys. 83 (1996) 955.

[6] P. Tomassini, Phys. Lett. B 411 (1997) 117.

[7] A. Berera and D. Hochberg, Phys. Rev. E 72 (2005) 057301.

[8] J. Zinn-Justin, Quantum Field Theory and Critical Phenomena, 4rth edition (Oxford University Press, Oxford, 2002).

[9] L.T. Adzhemyan, N.V. Antonov and A.N. Vasiliev, The Field Theoretic Renormalization Group in Fully Developed Turbulence (Gordon and Breach, New Your, 1999).

[10] P. Ramond, Field Theory: A Modern Primer (Benjamin-Cummings, Reading, Mass. 1981).

[11] D. Forster, D.R. Nelson and M.J. Stephen, Phys. Rev. A16 (1977) 732.

[12] W.D. McComb, Phys. Rev. E71 (2005) 037301.

[13] A. Berera and D. Hochberg, Phys. Rev. Lett. 99 (2007) 254501.

[14] P. Düben, D. Homeier, K. Jansen, D. Mesterhazy, G Münster and C. Urbach, DESY 08-133, MS-TP-08-21, arXiv:0809.4959 [hep-lat], September 2008.

[15] H.K. Janssen, Z. Phys. B 23 (1976) 377.

[16] R. Phythian, J. Phys. A 10 (1977) 777.

[17] R.D. Jensen, J. Stat. Phys. 25 (1981) 183.

[18] P.C. Martin, E.D. Siggia and H.A. Rose, Phys. Rev. A8 (1973) 423. 
[19] C. De Dominicis and L. Peliti, Phys. Rev. B18 (1978) 353.

[20] C. Becchi, A. Rouet and R. Stora, Phys. Lett. 52B (1974) 344 ; Ann. Phys. 98 (1976) 287.

[21] S.B. Pope, Turbulent Flows (Cambridge University Press, Cambridge, 2000).

[22] V.V. Lebedev and V.S. L'vov, Phys. Rev E 49 (1994) R959.

[23] E.V. Ivashkevich, J. Phys. A: Math. Gen. 30 (1997) L525.

[24] C. Tong, Physics of Fluids 15 (2003) 2073.

[25] M. Shah et al., in Proceedings of the Eurographics/ACM SIGGRAPH Symposium on Computer Animation, Grenoble, 2004, edited by R. Boulic and D.K. Pai (Eurographics Association, Aire-la-Ville, Switzerland, 2004), pp 213-220.

[26] S. Pokorski, Gauge Field Theories (Cambridge University Press, Cambridge 1987).

[27] J.D. Bjorken and S.D. Drell, Relativistic Quantum Fields (McGraw-Hill, New York, 1965).

[28] W.D. McComb, The Physics of Fluid Turbulence, (Oxford University Press, Oxford, 1990).

[29] S. Weinberg, Gravitation and Cosmology (Wiley, New York, 1972).

[30] M. Kardar, G. Parisi and Y.-C. Zhang, Phys. Rev. Lett. 58 (1987) 2087.

[31] E. Balkovsky, G. Falkovich, I. Kolokolov, and V. Lebedev, Phys. Rev. Lett. 78 (1997) 1452 ; Int. J. Mod. Phys. B 11 (1997) 3223.

[32] D. Homeier, K. Jansen, D. Mesterhazy and C. Urbach, Proc. of Path Integral-New Trends and Perspectives, Dresden, Germany, 2007, arXiv:0808.3481 [hep-lat].

[33] E.S. Abers and B.W. Lee, Phys. Lett. 9C (1973) 1-141. 